\title{
Recent Developments in Voltammetric Analysis of Pharmaceuticals Using Disposable Pencil Graphite Electrodes
}

\author{
Iulia Gabriela David ${ }^{1, *(1)}$, Mihaela Buleandra ${ }^{1}$, Dana Elena Popa ${ }^{1}$, Mihaela Carmen Cheregi ${ }^{1}\left(\mathbb{D}\right.$, Vasile David ${ }^{1}$, \\ Emilia Elena Iorgulescu $1, * \mathbb{C}$ and Georgiana Oana Tartareanu ${ }^{2}$
}

1 Department of Analytical Chemistry, Faculty of Chemistry, University of Bucharest, Panduri Av. 90-92, District 5, 050663 Bucharest, Romania; mihaela.buleandra@g.unibuc.ro (M.B.);

elena.popa@chimie.unibuc.ro (D.E.P.); mihaela.cheregi@g.unibuc.ro (M.C.C.); vasile.david@g.unibuc.ro (V.D.)

2 Department of Analytical Chemistry and Environmental Engineering, Faculty of Chemical Engineering and Biotechnologies, University Politehnica Bucharest, Gheorghe Polizu Street 1-7, District 1,

011061 Bucharest, Romania; oana.florea20@gmail.com

* Correspondence: gabrielaiulia.david@g.unibuc.ro (I.G.D.); emilia-elena.iorgulescu@chimie.unibuc.ro (E.E.I.)

check for

updates

Citation: David, I.G.; Buleandra, M.; Popa, D.E.; Cheregi, M.C.; David, V.; Iorgulescu, E.E.; Tartareanu, G.O.

Recent Developments in

Voltammetric Analysis of

Pharmaceuticals Using Disposable Pencil Graphite Electrodes. Processes 2022, 10, 472. https://doi.org/ $10.3390 /$ pr10030472

Academic Editor: Mihaela Puiu

Received: 10 February 2022

Accepted: 24 February 2022

Published: 26 February 2022

Publisher's Note: MDPI stays neutral with regard to jurisdictional claims in published maps and institutional affiliations.

Copyright: (C) 2022 by the authors. Licensee MDPI, Basel, Switzerland. This article is an open access article distributed under the terms and conditions of the Creative Commons Attribution (CC BY) license (https:// creativecommons.org/licenses/by/ $4.0 /)$.

\begin{abstract}
The even growing production of both well-known and new derivatives with pharmaceutical action involves the need for developing facile and reliable methods for the analysis of these compounds. Among the widely used instrumental techniques, the electrochemical ones are probably the simplest and the most rapid, also having good performance characteristics. However, the key tool in electroanalysis is the working electrode. Due to the inherent electrochemical and economic advantages of the pencil graphite electrode (PGE), the interest in its applicability in the analysis of different analytes has continuously increased in recent years. Thus, this paper aims to review the scientific reports published in the last 10 years on the use of the disposable eco- and user-friendly PGEs in the electroanalysis of compounds of pharmaceutical importance in different matrices. The PGE characteristics and designs (bare or modified with various types of materials), along with their applications and performance parameters (e.g., linear range, limit of detection, and reproducibility), will be discussed, and their advantages and limitations will be critically emphasized.
\end{abstract}

Keywords: voltammetry; pencil graphite electrode; pharmaceuticals

\section{Introduction}

In the current context of increasing drug use and abuse, pharmaceutical analysis is an important issue related to active ingredient identification and evaluation in raw materials, pharmaceutical formulations, and biological samples. It also involves impurities, degradation product, and excipient determination, content uniformity assessment, as well as dissolution and stability studies. Among the manifold applications that can be mentioned are drug quality control, therapeutic drug monitoring, and pharmacokinetic and clinical studies.

The pharmaceutical industry is one of the most regulated fields, with an increasing level of requirements set by the regulatory bodies. As a consequence, chemical analysts are continuously facing a challenge regarding the development, validation, and application of robust, fast, and cost-affordable analytical methods leading to reliable results, based on which decisions can be made. The pharmacopoeias [1] usually recommend chromatographic and spectrometric methods, but these conventional approaches present some shortcomings. Thus, despite the fact that the chromatographic techniques are the first choice for the separation of components from complex matrices, they require expensive equipment that needs to be carefully handled, tedious sample preparation steps, reagent consumption, and longer analysis time. Spectrometric methods are easy to use, offer (to a certain extent) economic value, and rely on noninvasive sample preparation and analysis procedures, but they suffer from a lack of selectivity (some analytes cannot be discriminated 
from the interferents absorbing at the same wavelength) and sensibility (sometimes, the necessity arises to concentrate or dilute the sample or the limited linear range due to deviations from the Beer's law) and are influenced by different factors (e.g., pH, temperature, and impurities) that can modify the sample absorption properties.

A promising approach in pharmaceutical analysis can be the use of electroanalytical techniques, especially due to the fact that they are suitable for miniaturization and portability, thus enabling on-site monitoring. Their efficiency was proven by their extensive use for the detection and quantification of various compounds, with powerful impacts on human health [2-5] and environment [6-8].

The electrochemical methods are versatile, non-destructive, cost-effective, fast, and facile, possess good analytical performance characteristics (e.g., precision, accuracy, selectivity, sensitivity, and large dynamic concentration range) [9], and do not involve timeconsuming pre-treatment processes [10]. In electrochemical techniques, qualitative information can be obtained by means of the analyte peak potential, while the peak current magnitude relates to the analyte concentration, being exploited for quantitative purposes. Moreover, the study of the target compound redox properties can lead to data regarding the in vivo metabolic processes taking place or the pharmacokinetic action [11].

The ability of the electrochemical methods to directly detect the target species in complex matrices is well known, but their analytical performances depend on the material of the working electrode. The limited employment of mercury electrodes due to environmental concerns and EU regulations, which restrict the use and storage of mercury [12], led to increased exploitation of metallic (e.g., gold, platinum, silver, palladium, and indium tin oxide) and carbon-based (e.g., glassy carbon, carbon paste, boron doped diamond, and graphite) substrates. One important drawback of the conventional solid electrodes is their surface fouling, a fact that seriously affects the possibility of obtaining a new surface with the same properties and, consequently, the electrode response reproducibility. To overcome this problem, the surface of the electrode must be mechanically or electrochemically cleaned [13], thus apearing to be a time-consuming step in the global analysis procedure. A simple alternative to electrode surface regeneration consists of using disposable electrodes like pencil graphite electrodes (PGEs).

PGEs consist of graphite leads of different diameters composed of graphite $(\sim 65 \%)$ as well as clay $(\sim 30 \%)$ and a binder (wax, resin, or high polymer), with the conductivity of this composite material being correlated with the clay/graphite ratio. Thus, B type leads are softer due to their higher graphite content, while a greater amount of clay in leads enhances the material hardness (H-type) [14]. The lead composition affects the structural and (electro)chemical properties of PGE. The graphite is responsible for the electrochemical activity, and the clay can act against electrode surface fouling [15]. The presence of sp2 hybridized carbon offers some special characteristics, such as good adsorption properties, electrical conductivity, and a small background current [16,17]. A recent paper [18] as well as our research (data not published) pointed out that polymer-based PGEs have superior electrochemical properties.

Commonly used in writing instruments, graphite pencil leads are commercially easily available and inexpensive, and they became a promising alternative as working electrodes in electroanalytical chemistry due to their recognized properties and advantages: a uniform lead surface, enlargeable active surface area, mechanical rigidity and stability, chemical inertness, portability, fast responses, and also being eco- and user-friendly $[14,19,20]$. It is worth emphasizing that a PGE is a disposable electrode, with the surface renewal being faster by simply replacing the pencil lead in comparison with polishing procedures applied to the classical solid electrodes [21]. Although another lead is used for every measurement, the reproducibility is assured by the composition uniformity, with the graphite leads being subjected to strict quality control during the fabrication process. Moreover, the same electrode surface area is always in contact with the analyzed solution.

Due to the large electrode surface area, which depends on how much the graphite lead is immersed into the solution, the target analytes can be detected at low concentrations. 
Additionally, the fact that PGEs can be exploited over a wide range of both negative and positive potentials extends the type and number of compounds that can be analyzed. The published studies proved that unmodified PGEs can successfully perform from $-1.20 \mathrm{~V}$ [22] to $+1.40 \mathrm{~V}$ vs. Ag/ $\mathrm{AgCl}[23]$.

By combining various voltametric techniques and PGEs as working electrodes, welldefined and reproducible signals are obtained which underlie reliable determinations. Moreover, the sensitivity and selectivity of the voltametric techniques can be improved by the working electrode modification, either electrochemical or chemical, that can also enhance the rate of the electron transfer processes.

Thus, bearing in mind the importance of pharmaceuticals in our day-to-day life and the inherent advantages of the voltametric methods and of the PGEs, this review seeks to provide an exhaustive analysis of the progress made in the last 10 years in the field of PGE applications in the voltametric determination of pharmaceutically important compounds. Due to the huge number of papers published on this topic, the present work refers only to the literature data appeared in the last 10 years, comprises only the voltametric techniques, and makes no reference to biosensors and DNA-based PGEs.

\section{PGE Manufacturing Procedures}

The most employed procedure for PGE preparation consists of a graphite pencil lead introduced into a mechanical pencil holder acting as support. In order to ensure a reproducible electroactive surface area, the same length of the pencil lead is always left outside the support and introduced in the voltametric solution. Electrical contact with the instrument is realized by means of a conductive wire which was soldered or wrapped to the metallic part of the mechanical pencil holder $[9,19,21,22,24-37]$ (Figure 1a). A micropipette tip [38], an insuline syringe [39], a glass cappilary [40,41], or a plastic [16,42-46] (Figure 1b) or Teflon tube [47-52] (Figure 1c,d) can also act as holder. An araldite mixture (epoxy resin, 1:1) [16,41,43-45], bentonite [38,39], or cyanoacrylate glue [40] was used to fix the graphite lead into the support. Other PGEs were obtained by covering a part of the graphite lead with nail polish [53] or with Teflon tape [20,54-65] (Figure 1e), leaving a constant length and area of nacked pencil mine to act as an electroative surface. The electrical contact with the potentiostat is provided by a metallic (copper) wire wrapped arround the end of the mine, which is not exposed to the analyzed solution. Ibrahim and Temerek [66] created a pencil holder consisting of a polypropylene tube having a heat-shrinking tube connected at the bottom to a metallic part, in which the graphite lead is fixed. Electrical contact is made by winding a copper wire on the metal portion.

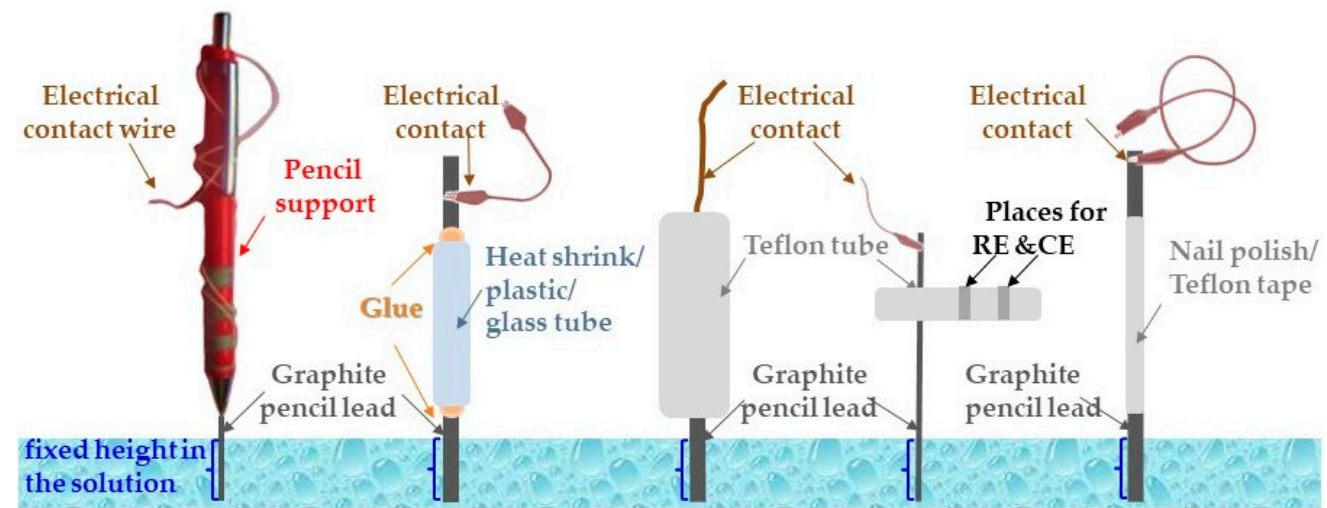

(a)

(b)

(c)

(d)

(e)

Figure 1. Schematic representation of the most commonly used types of PGEs with the graphite lead (a) fixed in a mechanical pencil, (b) inserted in a heat shrink, plastic, or glass tube and sealed with glue, (c,d) fixed in a Teflon tube, or (e) covered with nail polish or a Teflon band. 
The working electrode based on graphite leads with different hardness which is used for investigating the fouling effect during the voltammetry of the anesthetic propofol, a phenolic compound, is manufactured by peeling the two ends of wood pencils in order to expose the internal graphite mine. One end of the lead is used to provide the electrical contact with the potentiostat, whereas the other one constitutes the electroactive part of the electrode, which is cut to ensure a flat surface immersed in the investigated solution [15].

Sometimes, the graphite lead electrode is polished with an alumina slurry $[25,41,47,59,67]$, on sandpaper with different granulometrics $[40,42,68]$, on emery paper $[41,49-52,69]$ followed by a butter sheet [16,45], on butter paper [39], or on weighing paper $[20,57,58,62,70]$. Yen et al. [71] reported that amoxicicllin presented a better electrochemical response at the PGE mechanically treated with polishing paper in comparison with the original electrode.

Another approach for manufacturing procedures was reported by Tigari and Manjunatha [72] by preparing a paste electrode based on carbon nanotubes and 8B type pencil graphite powder composite modified with the anionic surfactant sodium lauryl sulphate for the electroanalysis of riboflavin in the presence of dopamine.

\section{Voltametric Performance Characteristics of PGEs vs. Other Common Bare Working Electrodes}

The performance characteristics of the three most popular and simplest carbon-based working electrodes, namely glassy carbon (GCE), pencil graphite (PGE), and carbon paste (CPE) electrodes, for methylergometrine maleate (MM) determination pointed out that the highest and best-defined oxidation signals were obtained for CPEs and PGEs, the lowest limit of detection (LoD) was attained for CPEs $(0.008 \mu \mathrm{g} / \mathrm{mL})$, and the widest linear range was recorded for GCEs $(0.50-5.50 \mu \mathrm{g} / \mathrm{mL})$, whereas the MM quantification for the PGEs enabled an LoD close to that obtained for CPEs and a linear range of one order of magnitude almost as large as that of the GCEs (Table 1) [24]. The two well-defined strontium ranelate irreversible anodic peaks recorded for GCEs, CPEs, and PGEs were exploited for its differential pulse (DP) and square wave (SW) voltametric determination. The PGEs provided the lowest LoD and limit of quantification (LoQ) values, while the widest linear ranges were obtained for the GCEs $(3.00-24.00 \mu \mathrm{g} / \mathrm{mL}$ and $2.50-25.00 \mu \mathrm{g} / \mathrm{mL}$ for both peaks by DPV and SWV, respectively) [25].

Itraconazole electro-oxidation and its adsorptive stripping (AdS) voltametric quantification were investigated at an ultra-trace graphite electrode (UTGE), PGE, and CPE. The analyte oxidation followed the same mechanism, and the linear ranges were comparable for all investigated electrodes, while the lowest LoDs $(8.40$ and $6.00 \mathrm{ng} / \mathrm{mL}$ by AdS-DPV and AdS-SWV, respectively) were attained for the CPE (Table 1) [26]. SWV determination of linezolid based on its irreversible diffusion-controlled oxidation was more sensitive for the PGE but within a narrower linear range (Table 1) in comparison with the CPE $\left(9.25 \times 10^{-9} \mathrm{~mol} / \mathrm{L}\right.$ and $2.96 \times 10^{-7}-2.22 \times 10^{-5} \mathrm{~mol} / \mathrm{L}$, respectively) [73]. The intensity of the dipyridamole and sulfamethoxazole DPV anodic signals decreased in the order PGE > GCE > Pt [27,28], whereas chloramphenicol [29] and famotidine [30] voltammograms recorded for the $\mathrm{Au}, \mathrm{Pt}, \mathrm{GCE}$, and PGE emphasized better defined and more intense signals when the PGE was the working electrode. Enhanced niclosamide [21], famotidine [31], amlodipine [39], piroxicam [42], and ketorolac [74] oxidation signals were obtained for the PGE vs. GCE.

As was already mentioned, pencil graphite is a composite material which has a different hardness depending on the graphite-to-clay or high polymer ratio. The electrochemical properties of the pencil graphite also depend on its composition. Our studies (unpublished data) showed that better and more intense voltametric signals were obtained at high polymer pencil leads, and the pencil lead hardness influenced the electrochemical behavior of the different analytes. Alves et al. [40] showed that $\mathrm{K}_{3}[\mathrm{Fe}(\mathrm{CN})]_{6}$ presented a sluggish electron transfer for $2 \mathrm{~B}$ type graphite leads, whereas in the B and HB mines, the redox peaks were intense and well-defined, with these signals being higher than those recorded in the same conditions for the GCE. On the other hand, the sensitivities $\left(\mathrm{A} \times \mathrm{L} / \mathrm{mol} \times \mathrm{cm}^{2}\right)$ 
of the dipyridamole, sulfamethoxazole DPV, and chloramphenicol cyclic voltammetric (CV) signals varied with the PGE hardness in the order $\mathrm{HB}>\mathrm{B}>\mathrm{H}>2 \mathrm{H}>2 \mathrm{~B}$ [27] and $\mathrm{B}>2 \mathrm{~B}>2 \mathrm{H}>\mathrm{H}>\mathrm{HB}[28]$ and $\mathrm{HB}>\mathrm{B}>\mathrm{H}>2 \mathrm{H}>2 \mathrm{~B}$ [29], respectively.

Kojabad and Ebrahimiasl [75] compared the effect of GCE and PGE modification with polypyrrole, CNT, and graphene on the tramadol DPV oxidation signal, with the highest peak current being provided by the CNT-modified electrodes. The results obtained for the two carbon-based electrodes used as substrates were similar, but due to the costeffectiveness, the authors selected PGEs for their further investigations.

Table 1. The performance characteristics of voltametric methods developed for drug quantitative determination for bare PGE.

\begin{tabular}{|c|c|c|c|c|c|c|}
\hline Analyte (Drug) & PGE Type & Technique & Linear Range (mol/L) & $\operatorname{LoD}(\mathrm{mol} / \mathrm{L})$ & Sample & Ref. \\
\hline Acyclovir & $2 B$ & DPV & $1.00 \times 10^{-6}-1.00 \times 10^{-4}$ & $3.00 \times 10^{-7}$ & Pharmaceutical tablets & [9] \\
\hline 4-Aminophenazone & $\mathrm{HB}$ & DPV & $1.00 \times 10^{-6}-1.00 \times 10^{-5}$ & $4.58 \times 10^{-8}$ & Plasma; urine & [76] \\
\hline Amlodipine & $\mathrm{HB}$ & $\begin{array}{l}\text { DPV } \\
\text { SWV }\end{array}$ & $\begin{array}{l}2.10 \times 10^{-9}-8.96 \times 10^{-8} \\
8.00 \times 10^{-10_{-5} .22} \times 10^{-8}\end{array}$ & $\begin{array}{l}4.00 \times 10^{-14} \\
2.00 \times 10^{-14}\end{array}$ & $\begin{array}{l}\text { Pharmaceutical tablets; spiked } \\
\text { human serum }\end{array}$ & [39] \\
\hline Amoxicicllin & - & DPV & $1.00 \times 10^{-6}-6.00 \times 10^{-5}$ & $2.24 \times 10^{-7}$ & - & [71] \\
\hline Cefidrin & - & SWV & $0.50-20.00 \mu \mathrm{g} / \mathrm{mL}$ & $0.15 \mu \mathrm{g} / \mathrm{mL}$ & $\begin{array}{l}\text { Pharmaceutical tablets } \\
\text { and powder }\end{array}$ & [23] \\
\hline Chloramphenicol & $2 \mathrm{H}$ & $\begin{array}{l}\text { LSV } \\
\text { SWV }\end{array}$ & $\begin{array}{l}2.50 \times 10^{-6}-1.00 \times 10^{-3} \\
2.50 \times 10^{-6}-7.50 \times 10^{-4}\end{array}$ & $\begin{array}{l}6.09 \times 10^{-7} \\
1.39 \times 10^{-6}\end{array}$ & Pharmaceutical capsules & [29] \\
\hline Chlorpromazine & $\mathrm{HB}$ & DPV & $1.00 \times 10^{-8}-8.00 \times 10^{-8}$ & $3.00 \times 10^{-9}$ & Pharmaceutical tablets & {$[16]$} \\
\hline Ciprofloxacin & $\mathrm{HB}$ & SWV & $1.20 \times 10^{-5}-5.50 \times 10^{-5}$ & $5.60 \times 10^{-6}$ & Pharmaceutical formulations & [40] \\
\hline Cysteamine & - & DPV & $1.00 \times 10^{-5}-1.10 \times 10^{-4}$ & $4.00 \times 10^{-6}$ & Urine & [54] \\
\hline Dipyridamole & $\mathrm{HB}$ & DPV & $5.00 \times 10^{-7}-2.50 \times 10^{-4}$ & $1.21 \times 10^{-7}$ & Pharmaceutical tablets & [27] \\
\hline Doxorubicin & - & $\begin{array}{l}\text { DPV } \\
\text { LSV }\end{array}$ & $\begin{array}{l}1.00 \times 10^{-5}-5.00 \times 10^{-5} \\
1.00 \times 10^{-5}-3.00 \times 10^{-5}\end{array}$ & $7.20 \times 10^{-6}$ & Serum & [70] \\
\hline Eugenol & $2 \mathrm{~B}$ & DPV & $3.00 \times 10^{-7}-5.00 \times 10^{-5}$ & $8.50 \times 10^{-8}$ & Pharmaceutical liquid & [32] \\
\hline Famotidine & $\mathrm{HB}$ & $\begin{array}{l}\text { SWV } \\
\text { DPV }\end{array}$ & $\begin{array}{l}5.00 \times 10^{-6}-1.80 \times 10^{-4} \\
4.72 \times 10^{-7}-4.95 \times 10^{-4}\end{array}$ & $\begin{array}{l}1.29 \times 10^{-6} \\
1.51 \times 10^{-7}\end{array}$ & Pharmaceutical tablets & $\begin{array}{l}{[30]} \\
{[31]}\end{array}$ \\
\hline $\begin{array}{l}\text { Flutamide } \\
\text { Irinotecan }\end{array}$ & $2 \mathrm{~B}$ & CAdS-SWV & $\begin{array}{l}3.98 \times 10^{-7}-6.36 \times 10^{-6} \\
7.94 \times 10^{-8}-4.03 \times 10^{-7}\end{array}$ & $\begin{array}{l}1.55 \times 10^{-8} \\
1.68 \times 10^{-9}\end{array}$ & Serum; urine & [22] \\
\hline Hydroxyurea & - & & $1.00 \times 10^{-5}-1.00 \times 10^{-3}$ & $7.89 \times 10^{-6}$ & Pharmaceutical tablets; urine & [11] \\
\hline Imipenem & & $\begin{array}{l}\mathrm{Tb}^{3+} \text {-complex } \\
\text { deposition } \\
+\mathrm{CV}\end{array}$ & $1.50 \times 10^{-5}-7.00 \times 10^{-5}$ & $2.44 \times 10^{-6}$ & $\begin{array}{l}\text { Pharmaceutical vials; } \\
\text { rabbit plasma }\end{array}$ & [77] \\
\hline Itraconazole & $\mathrm{HB}$ & $\begin{array}{l}\text { AdS-DPV } \\
\text { AdS-SWV }\end{array}$ & $\begin{array}{c}32.00-169.00 \\
10.60-127.00 \mathrm{ng} / \mathrm{mL}\end{array}$ & $\begin{array}{c}9.10 \\
8.70 \mathrm{ng} / \mathrm{mL}\end{array}$ & $\begin{array}{l}\text { Pharmaceutical capsules; } \\
\text { serum; urine }\end{array}$ & [26] \\
\hline Linezolid & - & SWV & $2.96 \times 10^{-8}-5.93 \times 10^{-7}$ & $1.39 \times 10^{-9}$ & $\begin{array}{l}\text { Pharmaceutical and } \\
\text { biological samples }\end{array}$ & [73] \\
\hline $\begin{array}{l}\text { Methylergometrine } \\
\text { maleate }\end{array}$ & $\mathrm{HB}$ & DPV & $0.10-1.00 \mu \mathrm{g} / \mathrm{mL}$ & $0.02 \mu \mathrm{g} / \mathrm{mL}$ & $\begin{array}{l}\text { Pharmaceutical tablets } \\
\text { and ampoules }\end{array}$ & [24] \\
\hline Niclosamide & $2 \mathrm{~B}$ & DPV & $5.00 \times 10^{-7}-1.00 \times 10^{-5}$ & $1.50 \times 10^{-9}$ & Pharmaceutical tablets & [21] \\
\hline Paclitaxel & HB & DPV & $4.00 \times 10^{-7}-3.00 \times 10^{-6}$ & $2.46 \times 10^{-9}$ & $\begin{array}{l}\text { Pharmaceutical injections; } \\
\text { plasma; urine }\end{array}$ & [78] \\
\hline Piroxicam & $4 \mathrm{~B}$ & DPV & $2.91 \times 10^{-5}-1.63 \times 10^{-4}$ & $2.10 \times 10^{-6}$ & Pharmaceutical capsules & [42] \\
\hline Strontium Ranelate & HB & $\begin{array}{l}\text { DPV } \\
\text { SWV }\end{array}$ & $\begin{array}{l}1.00-10.00 \mu \mathrm{g} / \mathrm{mL} \\
1.00-10.00 \mu \mathrm{g} / \mathrm{mL}\end{array}$ & $\begin{array}{l}0.17 \mu \mathrm{g} / \mathrm{mL} \\
0.19 \mu \mathrm{g} / \mathrm{mL}\end{array}$ & Pharmaceutical formulations & [25] \\
\hline Sulfamethoxazole & B & DPV & $1.00 \times 10^{-5}-2.50 \times 10^{-4}$ & $4.04 \times 10^{-6}$ & Pharmaceutical tablets & [28] \\
\hline Vildagliptin & $2 \mathrm{~B}$ & SWV & $2.94 \times 10^{-6}-4.99 \times 10^{-5}$ & $8.20 \times 10^{-8}$ & Pharmaceutical tablets; urine & [13] \\
\hline $\begin{array}{l}\text { Vitamin B1 } \\
\text { Vitamin B6 }\end{array}$ & $\mathrm{HB}$ & DPV & $\begin{array}{l}1.00 \times 10^{-5}-1.00 \times 10^{-3} \\
5.00 \times 10^{-6}-1.00 \times 10^{-3}\end{array}$ & $\begin{array}{l}5.34 \times 10^{-6} \\
2.81 \times 10^{-6}\end{array}$ & Pharmaceutical vials & [33] \\
\hline $\begin{array}{l}\text { Vitamin B2 } \\
\text { Vitamin B6 }\end{array}$ & HB & SWV & $\begin{array}{l}1.00 \times 10^{-7}-7.50 \times 10^{-4} \\
2.50 \times 10^{-5}-2.50 \times 10^{-3}\end{array}$ & $\begin{array}{l}7.38 \times 10^{-8} \\
1.10 \times 10^{-5}\end{array}$ & Pharmaceutical tablets & [79] \\
\hline Vitamin B12 & - & DPV & $2.50 \times 10^{-9}-3.00 \times 10^{-8}$ & $8.20 \times 10^{-8}$ & Pharmaceutical tablets & [55] \\
\hline
\end{tabular}

DPV = differential pulse voltammetry; SWV = square wave voltammetry; LSV = linear sweep voltammetry; CAdSSWV = cathodic adsorptive stripping square wave voltammetry; CV = cyclic voltammetry; AdS-DPV = adsorptive stripping differential pulse voltammetry; AdS-SWV = adsorptive stripping square wave voltammetry. 


\section{Voltametric Analysis of Pharmaceutical Active Compounds Using PGEs}

\subsection{Applications of Unmodified PGEs to the Voltametric Analysis of Pharmaceutical Compounds}

Considering all the above mentioned advantages offered by the use of PGEs as working electrodes in electrochemical analysis, numerous examples of research describe their applicability in the voltametric determination of various drugs, either individual or in mixtures, from very different matrices, like pharmaceuticals, biological fluids, or food or water samples. The literature data reported in the last 10 years regarding the applications of unmodified PGEs in this field are summarized in Table 1. The main benefits of employing a bare PGE are, beside its disposability, the simplicity and ease of use, time, and reagent consuming steps being absent. It can be observed from Table 1 that the LoD obtained for this working electrode are below the micromolar level, highlighting the good sensitivity of the bare PGEs, which makes the methods using them appropriate for the drug quantification in the selected samples.

\subsection{Applications of Electrochemically Pretreated PGEs to the Voltametric Analysis of Pharmaceutical Compounds}

The performance parameters (i.e., sensitivity and selectivity) of solid working electrodes can be improved by the modification of their electroactive surface area. The simplest, easiest, and most rapid way to change the structure and morphology of the carbon-based electrode surface, and thus of the PGE, with the use of minimum amounts of non-toxic reagents [80] (usually only a supporting electrolyte is required) is electrochemical pretreatment, which consists of potential cycling or electrochemical anodization [10]. During this process, the electrode surface is cleaned, and new active edge-plane sites are produced along with the generation of oxygen-containing moieties (e.g., carbonyl, carboxyl, and epoxide) $[14,62,80,81]$. These functional groups activate the electrode surface by enhancing its hydrophilic properties [82] and may have an electrocatalytic effect, improving the electron transfer rate for some analytes [83]. The PGE pretreatment reduces the background current [74], and due to the oxidation of the graphite layer, the effective surface area of the electrode is increased [84] by the formation of carbon nanoparticles [81], thus offering better sensitivity and more reproducible results. All these observations were supported by experimental results. Thus, scanning electron microscopy (SEM) images emphasized that the non-treated PGE had a smooth surface, whereas the electrochemical pretreated PGE (ET-PGE) presented porosity and an increased surface area [80]. CV investigations pointed out the increase in the ET-PGE effective electroactive surface area in comparison with the PGE (e.g., $0.0170 \mathrm{~cm}^{2}$ for an HB type PGE and $0.0235 \mathrm{~cm}^{2}$ for ET-PGE [43], $0.0355 \mathrm{~cm}^{2}$ for a 2B type PGE and $0.1408 \mathrm{~cm}^{2}$ for ET-PGE [56], or approximately a threefold increase [80]). The electrochemical impedance spectroscopic (EIS) studies emphasized a lower charge transfer resistance and thus a higher conductivity of the ET-PGE $[56,80]$ due to good electrocatalytic activity and a high interfacial electron transfer rate at the ET-PGE, resulting in enhanced peak currents.

The analytes' voltametric behavior depends on the PGE's electrochemical treatment (activation) method (at a constant current [62], performed by maintaining the working electrode for a given time at a certain potential [36,37,82,84-88], or potentiodynamic, being carried out by CV $[20,60,68,89-92]$ ) as well as on the instrumental parameters (pretreatment time and potential in the potentiostatic procedure and scan rate and potential range in the $\mathrm{CV}$ method) and on the nature, concentration, and $\mathrm{pH}$ of the supporting electrolyte $[14,83]$. For example, in the case of diclofenac sodium, the potentiostatic activation resulted in a more pronounced enhancement of the DPV anodic peak in comparison with electroactivation by $\mathrm{CV}$ [80]. For the sensitive and selective determination of paracetamol in the presence of ascorbic acid and caffeine, Kyun et al. [81] performed PGE electrochemical treatment using different acids $\left(\mathrm{HCl}, \mathrm{HNO}_{3}\right.$, and $\mathrm{H}_{2} \mathrm{SO}_{4}$ at various concentrations from 0.10 to $5.00 \mathrm{~mol} / \mathrm{L}$ ) and varying the number of cyclic voltametric scans (from 5 to 50 ). The best results were obtained using $0.50 \mathrm{~mol} / \mathrm{L} \mathrm{H}_{2} \mathrm{SO}_{4}$ as the optimized activation medium and 10 cycles of the potential in the range $0.00-2.10 \mathrm{~V}$. 
Moreover, by applying chronoamperometry in $\mathrm{H}_{3} \mathrm{PO}_{4}$, Dokur et al. [10] obtained a phosphorous-doped PGE with improved performances for pyridoxine voltametric analysis, whereas the potentiostatic treatment of a PGE in a melamine and $\left(\mathrm{NH}_{4}\right)_{2} \mathrm{SO}_{4}$ mixture resulted in the deposition of a sulfur and nitrogen co-doped graphene nanocomposite on the PGE [63].

In some research, the electroactivated PGE was applied as such in the pharmaceutical electrochemical analysis (Table 2), while in other research, its surface was further modified [36,37,68,85,86,91,92] (Table 3).

Table 2. The performance characteristics of voltametric methods developed for drug quantitative determination at ET-PGE.

\begin{tabular}{|c|c|c|c|c|c|c|}
\hline Analyte (Drug) & PGE Type/ET Conditions & $\begin{array}{l}\text { Analysis } \\
\text { Technique }\end{array}$ & Linear Range (mol/L) & $\begin{array}{c}\text { LoD } \\
(\mathrm{mol} / \mathrm{L})\end{array}$ & Sample & Ref. \\
\hline $\begin{array}{l}\text { Ascorbic acid } \\
\text { Norepinephrine } \\
\text { Uric acid }\end{array}$ & $1.40 \mathrm{~V}, 60 \mathrm{~s}$ & DPV & $\begin{array}{l}1.00 \times 10^{-7}-8.00 \times 10^{-7} \\
2.00 \times 10^{-8}-1.70 \times 10^{-7} \\
4.00 \times 10^{-8}-1.75 \times 10^{-7}\end{array}$ & $\begin{array}{l}2.70 \times 10^{-8} \\
4.00 \times 10^{-9} \\
1.00 \times 10^{-8}\end{array}$ & $\begin{array}{l}\text { Pharmaceutical } \\
\text { formulations; urine }\end{array}$ & [84] \\
\hline $\begin{array}{l}\text { Diclofenac } \\
\text { sodium }\end{array}$ & $\begin{array}{c}\mathrm{H} /-0.50 \mathrm{~V}, 300 \mathrm{~s} \\
0.50 \mathrm{~mol} / \mathrm{L} \mathrm{ABS} \\
\mathrm{pH} 4.80+0.10 \mathrm{~mol} / \mathrm{L} \mathrm{NaCl}\end{array}$ & DPV & $2.30 \times 10^{-7}-1.29 \times 10^{-5}$ & $1.20 \times 10^{-7}$ & $\begin{array}{l}\text { Pharmaceutical } \\
\text { tablets; urine }\end{array}$ & [80] \\
\hline Methamphetamine & $\begin{array}{c}1.70 \mathrm{~V}, 10 \mathrm{~min} ; 0.10 \mathrm{~mol} / \mathrm{L} \\
\text { BRB pH } 11.00\end{array}$ & DPV & $7.40 \times 10^{-8}-5.40 \times 10^{-5}$ & $5.00 \times 10^{-8}$ & Serum; urine & [93] \\
\hline Morphine & $\begin{array}{c}+1.80 \mathrm{~V}, 300 \mathrm{~s} ; 0.50 \mathrm{~mol} / \mathrm{L} \\
\text { PBS pH } 7.00\end{array}$ & $\begin{array}{l}\text { DPV } \\
\text { Amp }\end{array}$ & $1.00 \times 10^{-6}-1.00 \times 10^{-4}$ & $2.60 \times 10^{-7}$ & Street drugs; urine & [94] \\
\hline Pantoprazole & $\begin{array}{l}\mathrm{HB} /+1.30 \mathrm{~V}, 30 \mathrm{~s} \\
\mathrm{BRB} \text { pH } 7.00\end{array}$ & $\begin{array}{c}\mathrm{Co}^{2+}- \\
\text { complexation }+ \\
\text { AdS-SWV }\end{array}$ & $1.00 \times 10^{-10}-9.00 \times 10^{-9}$ & $4.00 \times 10^{-11}$ & $\begin{array}{l}\text { Pharmaceutical } \\
\text { tablets and vials; } \\
\text { spiked rabbit } \\
\text { plasma }\end{array}$ & [82] \\
\hline Vitamin B6 & $\begin{array}{c}2.00 \mathrm{~V}, 100 \mathrm{~s} ; 0.10 \mathrm{~mol} / \mathrm{L} \\
\mathrm{H}_{3} \mathrm{PO}_{4}\end{array}$ & DPV & $5.00 \times 10^{-7}-3.00 \times 10^{-4}$ & $2.19 \times 10^{-7}$ & $\begin{array}{l}\text { Energy drink; } \\
\text { vitamin water }\end{array}$ & {$[10]$} \\
\hline Acetaminophen & $\begin{array}{c}\mathrm{HB} / \mathrm{CV}: 0.00-2.10 \mathrm{~V} ; \mathrm{n}=10 \\
0.50 \mathrm{~mol} / \mathrm{L} \mathrm{H}_{2} \mathrm{SO}_{4}\end{array}$ & DPV & $1.00 \times 10^{-6}-1.00 \times 10^{-3}$ & $1.74 \times 10^{-7}$ & $\begin{array}{c}\text { Pharmaceutical } \\
\text { tablets }\end{array}$ & [81] \\
\hline Albendazole & $\begin{array}{c}\mathrm{HB} / \mathrm{CV}:-2.00 \text { to } 2.00 \mathrm{~V} \\
\mathrm{n}=5 ; 0.05 \mathrm{~V} / \mathrm{s} ; 0.10 \mathrm{M} \mathrm{HCl}\end{array}$ & DPV & $2.50 \times 10^{-4}-1.45 \times 10^{-3}$ & $5.42 \times 10^{-9}$ & $\begin{array}{l}\text { Pharmaceutical } \\
\text { tablets; urine }\end{array}$ & [95] \\
\hline $\begin{array}{c}\text { Epinephrine } \\
\text { Norepinephrine } \\
\text { Epinephrine } \\
\text { Norepinephrine } \\
\end{array}$ & $\begin{array}{c}\mathrm{HB} / \mathrm{CV}:-0.20 \text { to } 2.00 \mathrm{~V} \\
\mathrm{n}=10 ; 0.50 \mathrm{~V} / \mathrm{s} \\
\mathrm{BRB} \mathrm{pH} 2.21\end{array}$ & $\begin{array}{l}\text { CSWV } \\
\text { SWV }\end{array}$ & $\begin{array}{l}2.50 \times 10^{-6}-2.50 \times 10^{-4} \\
2.50 \times 10^{-6}-2.50 \times 10^{-4} \\
2.50 \times 10^{-6}-2.50 \times 10^{-4} \\
2.50 \times 10^{-6}-2.40 \times 10^{-4}\end{array}$ & $\begin{array}{l}8.32 \times 10^{-7} \\
9.92 \times 10^{-7} \\
1.46 \times 10^{-6} \\
1.46 \times 10^{-6}\end{array}$ & $\begin{array}{l}\text { Pharmaceutical } \\
\text { vials; plasma } \\
\text { Human plasma }\end{array}$ & $\begin{array}{l}{[96,} \\
97]\end{array}$ \\
\hline Hydrochlorthiazide & $\begin{array}{c}\mathrm{HB} / \mathrm{CV}: 0.40 \text { to } 1.20 \mathrm{~V} ; \\
\mathrm{n}=50 ; 0.10 \mathrm{~V} / \mathrm{s} ; 0.10 \mathrm{~mol} / \mathrm{L} \\
\mathrm{PBS} \mathrm{pH} 7.00\end{array}$ & $\begin{array}{l}\text { DPV } \\
\text { SWV }\end{array}$ & $\begin{array}{l}4.00 \times 10^{-6}-1.40 \times 10^{-4} \\
1.00 \times 10^{-6}-2.00 \times 10^{-5}\end{array}$ & $\begin{array}{l}3.25 \times 10^{-6} \\
4.21 \times 10^{-7}\end{array}$ & $\begin{array}{l}\text { Pharmaceutical } \\
\text { tablets; urine }\end{array}$ & [43] \\
\hline Ketorolac & $\begin{array}{l}\text { CV: } 0.60 \text { to } 1.60 \mathrm{~V} ; \mathrm{n}=10 ; \\
0.20 \mathrm{~mol} / \mathrm{L} \text { PBS } \mathrm{pH} 3.00\end{array}$ & DPV & $2.00 \times 10^{-6}-1.00 \times 10^{-3}$ & $4.59 \times 10^{-7}$ & $\begin{array}{l}\text { Pharmaceutical } \\
\text { tablets; urine }\end{array}$ & {$[74]$} \\
\hline Lamotrigine & $\begin{array}{c}\mathrm{HB} / \mathrm{CV}:-0.20 \text { to } 2.00 \mathrm{~V} \\
\mathrm{n}=10 ; 0.50 \mathrm{~V} / \mathrm{s} \\
\mathrm{BRB} \text { pH } 2.21\end{array}$ & LSV & $2.50 \times 10^{-5}-1.00 \times 10^{-3}$ & $1.94 \times 10^{-5}$ & $\begin{array}{l}\text { Pharmaceutical } \\
\text { tablets }\end{array}$ & [98] \\
\hline Rapamycin & $\begin{array}{c}\text { 2B/CV: } 1.50 \text { to } 2.00 \mathrm{~V} ; \\
\mathrm{n}=50 ; 0.05 \mathrm{~V} / \mathrm{s} ; 0.10 \mathrm{~mol} / \mathrm{L} \\
\text { PBS pH } 7.00\end{array}$ & DPV & $1.00 \times 10^{-8}-2.50 \times 10^{-4}$ & $7.50 \times 10^{-9}$ & $\begin{array}{l}\text { Pharmaceutical } \\
\text { tablets }\end{array}$ & [56] \\
\hline
\end{tabular}

ABS = acetate buffer solution; BRB = Britton-Robinson buffer; PBS = phosphate buffer solution; CSWV = cyclic square wave voltammetry; Amp = amperometry.

\subsection{Applications of (Electro)chemically Modified PGEs to the Voltametric Analysis of Pharmaceutical Compounds}

It is well established that modified electrodes provide wider linear ranges, lower limits of detection, better selectivity, high precision, and good accuracy of the electrochemical measurements. In order to obtain one or more of these improved sensing performance characteristics, the electrodes' electroactive surfaces are changed by covering them with proper selected species. Nanomaterials and polymers deposited at the PGE's surface usually have an electrocatalytic effect and facilitate the electron transfer between the electrode and the electroactive species, increase the effective electroactive surface area, and reduce electrode fouling. As can be observed from Table 3, the most frequently used modifiers, immobilized on the PGE surface by various physical or chemical methods, 
are nanomaterials like nanoparticles (including magnetic nanoparticles [99]), nanofibers, and nanodots based on carbon (e.g., carbon nanotubes or graphene [100]), metals (noble metals, $\mathrm{Cu}$, etc. or bimetallic structures), metaloxides $\left(\mathrm{Mn}_{5} \mathrm{O}_{8}, \mathrm{MnO}_{2}, \mathrm{TiO}_{2}\right.$, etc.) or metal complex structures [101], polymers and molecularly imprinted polymers (MIP) [102], or combinations of them, resulting in hybrid nanomaterial composites.

Thin metallic films and metal or metallic oxide micro- or nanoparticles have excellent electric and catalytic properties, and therefore, they are widely used to improve the electrochemical performances of solid electrodes, also making them more suitable for analysis in the cathodic range. Despite the fact that this type of modified electrode contains a small amount of metal, it possesses the advantages of the corresponding metallic electrodes and is adequate for stripping analysis. Thus, based on its reduction signal from $-0.93 \mathrm{~V}$, the antineoplastic drug lomustine was quantified as a PGE in situ electrochemically modified in a single step with a thin mercury film [103] (Table 3). The in situ coating has the advantage of eliminating the electrode preparation steps before analysis, but it has the drawback of introducing the modifier in the sample solution, making the solution inadequate for further investigations. A bismuth film-plated PGE [104] and a porous copper microparticles modified PGE were fabricated by ex situ potentiostatic deposition [19] and then transferred to the analyte solution, which was voltametrically investigated (Table 3). Layers and nanoparticles of various other materials were also deposited on the PGE surface by applying a given potential for a certain time [61,63-65,68,91,92,105-107].

An easy way to cover the PGE surface is to apply multiple CV scans to a solution containing the desired modifier(s) $[20,60,86,87,108]$, most often resulting in electrode surface polymeric films like polypyrrole [86,109,110], polyaniline [105,109], poly(2-thiobarbituric acid) [59], poly(Azure-B) [45], poly(xylenol orange) [46], poly(eriochrom black T) [111, 112], poly(bromocresol green) [113], etc. or MIPs if the polymerization solutions contain both the monomer(s) and the analyte(s), which act as template molecule(s) $[38,49,89,91,92,106,107,114-124]$ (Table 3). Covering an electrode with electrogenerated polymeric films is simple, rapid, and cost-effective and results in sensors with enhanced electrochemical performance characteristics. The electrocatalytic properties of the modified PGE depends on the polymeric film thickness and stability, with the optimum ones being established by adjusting the electrochemical parameters (e.g., deposition potential and time, scan rate, and number of multiple voltametric cycles).

In the case of MIP, after the polymerization step, the template (analyte) inserted into the polymeric matrix must be retrieved in order to create the size- and shape-specific cavities responsible for the selective recognition of the analyte. The encapsulated template molecules were extracted by introducing the polymer adduct into an adequate solvent or solvent mixture for a given time, usually under stirring $[36,37,48-52,65,91,92,120,123,125-128]$, with the complete removal of the analyte molecules most often being confirmed by the absence of the characteristic signal in voltametric recordings $[48,50-52,120,123,125]$ but also by FTIR [52], UV-Vis [48,49], or EDS analysis [120,128]. Some studies reported the electrochemical de-doping of the imprinted polymer performed either by cyclic [129] or differential pulse potential scanning $[106,107,121,122,124]$ or by applying to the PGE covered with the polymeric film a given potential for a well-established time period [38].

In some cases, electrodeposition (e.g., AuNPs) and electropolymerization (e.g., polypyrrole [37], and poly(L-methionine [130]) on the PGE surface were achieved in one single step by $\mathrm{CV}$, with other modification procedures comprising both cyclic voltametric and potentiostatic steps [53,91,129,131].

Other modified PGEs were manufactured by dip $[17,61,69,87,88,90,92,127,132,133]$ or spin coating $[48,50-52,125,126,134]$. Drop coating was applied to produce $\mathrm{Mo}_{5} \mathrm{O}_{8} /$ PGE [34], Trypan blue-modified PGEs [44], different CNT-modified PGEs [47,57,58,68], or PGEs covered with various modifiers $[41,49,62,65,135]$. After that, the modified PGE was dried either in air $[35,48,52,66,68,69,90,136]$ or in other well-established conditions (e.g., in a desiccator [50] or at a certain temperature [125,137]). The substrate could be either inactivated or electrochemically treated (ET) PGEs (Table 3). 
Some PGEs were modified with several species combining two or more of the abovementioned surface covering methods [92]. For example, the nitrogen-doped graphene (NDG) was electrochemically synthesized and deposited on the PGE in a single step at room temperature by immersing the PGE in a graphene oxide (GO) in an $\mathrm{NH}_{4} \mathrm{Cl} / \mathrm{NH}_{3}$ suspension and applying a potential of $-1.20 \mathrm{~V}$ for $300 \mathrm{~s}$, resulting in an NDG/PGE, which was further immersed for $30 \mathrm{~min}$ in the MIP based on poly(acrylamide) (PAA)/sol-gel to obtain an NDG/MIP(PAA)/sol-gel/PGE [127]. MIP based on a poly(methacrylic acid)/solgel/phosphotungstic acid/reduced GO (MIP(PMA)/sol-gel/PWA/rGO)-modified PGE was prepared by dropping the MIP on the surface of a PWA/rGO/PGE obtained previously by potentiostatic deposition of the $\mathrm{PWA} / \mathrm{rGO}$ composite [65]. In the doublepolymer (PVC/PEDOT-C14)-modified PGE, the conductive polymer constituting C14 alkyl group derivatized poly(3,4-ethylenedioxythiophene) (PEDOT-C14) was obtained by cyclic voltametric electropolymerization of the monomer 2-n-tetradecyl-2,3-dihydro-thieno [3,4-b][1,4]dioxine (EDOT-C14), resulting in a PEDOT- C $_{14} /$ PGE, which was subsequently dip-coated with a plasticized polyvinyl chloride (PVC) membrane [138].

Table 3. The performance characteristics of voltametric methods developed for drug quantitative determination at (electro)chemically modified PGE.

\begin{tabular}{|c|c|c|c|c|c|c|}
\hline Analyte (Drug) & $\begin{array}{c}\text { PGE } \\
\text { Type/Modifier } \\
\end{array}$ & $\begin{array}{c}\text { Analysis } \\
\text { Technique }\end{array}$ & Linear Range (mol/L) & LoD (mol/L) & Sample & Ref. \\
\hline Lomustine & 2B/Hg film & CAdS-SWV & $1.92 \times 10^{-7}-1.36 \times 10^{-5}$ & $8.13 \times 10^{-8}$ & $\begin{array}{l}\text { Human blood } \\
\text { and urine }\end{array}$ & [103] \\
\hline Diazepam & ET/Bi film & DPV & $1.40 \times 10^{-6}-1.067 \times 10^{-5}$ & $1.10 \times 10^{-6}$ & $\begin{array}{l}\text { Pharmaceutical } \\
\text { tablets; human urine }\end{array}$ & [139] \\
\hline Metronidazole & -/Bi film & $\begin{array}{l}\text { CV } \\
\text { DPV } \\
\text { Amp }\end{array}$ & $\begin{array}{l}3.00 \times 10^{-7}-8.00 \times 10^{-5} \\
2.00 \times 10^{-7}-3.00 \times 10^{-5} \\
1.00 \times 10^{-6}-1.10 \times 10^{-5}\end{array}$ & $\begin{array}{l}3.90 \times 10^{-8} \\
2.94 \times 10^{-6}\end{array}$ & $\begin{array}{l}\text { Pharmaceutical } \\
\text { tablets; human } \\
\text { serum }\end{array}$ & [104] \\
\hline $\begin{array}{l}\text { Flutamide } \\
\text { Cyproterone } \\
\text { acetate }\end{array}$ & $\begin{array}{l}\text { 2B/nanoacetylene } \\
\text { black }\end{array}$ & CAdS-SWV & $\begin{array}{l}2.60 \times 10^{-8}-4.77 \times 10^{-7} \\
6.60 \times 10^{-8}-1.10 \times 10^{-6}\end{array}$ & $\begin{array}{l}1.39 \times 10^{-9} \\
4.74 \times 10^{-9}\end{array}$ & $\begin{array}{l}\text { Pharmaceutical } \\
\text { tablets; human } \\
\text { serum and urine }\end{array}$ & [66] \\
\hline Elbasivir & $\mathrm{H} / \mathrm{Mn}_{5} \mathrm{O}_{8} \mathrm{NPs}$ & SWV & $2.00 \times 10^{-7}-3.00 \times 10^{-6}$ & $4.00 \times 10^{-8}$ & $\begin{array}{l}\text { Spiked human } \\
\text { plasma }\end{array}$ & [34] \\
\hline Ledipasivir & $\mathrm{H} / \mathrm{MnO}_{2} \mathrm{NPs}$ & SWV & $2.50 \times 10^{-8}-3.60 \times 10^{-6}$ & $5.10 \times 10^{-9}$ & $\begin{array}{l}\text { Pharmaceutical } \\
\text { tablets; rat plasma }\end{array}$ & [35] \\
\hline Valacyclovir & $\mathrm{H} / \mathrm{CuMPs}$ & AdS-SWV & $2.00 \times 10^{-9}-1.00 \times 10^{-8}$ & $1.78 \times 10^{-10}$ & $\begin{array}{l}\text { Pharmaceutical } \\
\text { tablets }\end{array}$ & [19] \\
\hline Aceclofenac & 2B/MWCNTs & DPV & $1.00 \times 10^{-6}-6.00 \times 10^{-5}$ & $2.60 \times 10^{-9}$ & $\begin{array}{l}\text { Pharmaceutical } \\
\text { tablets; human urine }\end{array}$ & [47] \\
\hline Buprenorphine & ET/MWCNTs & DPV & $\begin{array}{l}1.00 \times 10^{-12}-1.09 \times 10^{-10} \\
1.09 \times 10^{-10}-1.10 \times 10^{-7}\end{array}$ & $6.00 \times 10^{-13}$ & $\begin{array}{l}\text { Human plasma } \\
\text { and urine }\end{array}$ & [137] \\
\hline $\begin{array}{l}\text { Diclofenac } \\
\text { sodium }\end{array}$ & $\mathrm{H} / \mathrm{MWCNTs}$ & DPV & $4.70 \times 10^{-8}-1.29 \times 10^{-5}$ & $1.70 \times 10^{-8}$ & $\begin{array}{c}\text { Pharmaceutical } \\
\text { tablets; human urine }\end{array}$ & [57] \\
\hline Methadone & $\mathrm{H} / \mathrm{MWCNTs}$ & DPV & $1.00 \times 10^{-7}-1.50 \times 10^{-5}$ & $8.70 \times 10^{-8}$ & $\begin{array}{l}\text { Human serum } \\
\text { and urine }\end{array}$ & [58] \\
\hline Hydrochlorthiazide & $\mathrm{HB} / \mathrm{TyB}$ & $\begin{array}{l}\text { DPV } \\
\text { SWV }\end{array}$ & $\begin{array}{l}5.00 \times 10^{-7}-7.00 \times 10^{-6} \\
1.00 \times 10^{-7}-5.00 \times 10^{-6}\end{array}$ & $\begin{array}{l}1.33 \times 10^{-7} \\
3.20 \times 10^{-8}\end{array}$ & $\begin{array}{l}\text { Pharmaceutical } \\
\text { tablets }\end{array}$ & [44] \\
\hline $\begin{array}{l}\text { Daclatasivir } \\
\text { Vardenafil }\end{array}$ & $\mathrm{H} / \mathrm{PXO}$ & SWV & $\begin{array}{l}2.00 \times 10^{-7}-7.00 \times 10^{-7} \\
1.00 \times 10^{-7}-6.00 \times 10^{-7}\end{array}$ & $\begin{array}{l}3.00 \times 10^{-8} \\
2.00 \times 10^{-8} \\
\end{array}$ & Rabbit plasma & [46] \\
\hline $\begin{array}{l}\text { Domperidone } \\
\text { Rabeprazole } \\
\text { sodium }\end{array}$ & -/PEBT & $\begin{array}{c}C V \\
\text { AdS-SWV } \\
C V \\
\text { AdS-SWV }\end{array}$ & $\begin{array}{l}5.20 \times 10^{-6}-9.00 \times 10^{-5} \\
5.00 \times 10^{-7}-8.00 \times 10^{-6} \\
4.10 \times 10^{-6}-1.20 \times 10^{-4} \\
7.50 \times 10^{-7}-8.00 \times 10^{-6}\end{array}$ & $\begin{array}{l}5.20 \times 10^{-6} \\
5.00 \times 10^{-7} \\
4.10 \times 10^{-6} \\
7.50 \times 10^{-6}\end{array}$ & $\begin{array}{l}\text { Synthetic mixture; } \\
\text { human plasma }\end{array}$ & [111] \\
\hline
\end{tabular}


Table 3. Cont.

\begin{tabular}{|c|c|c|c|c|c|c|}
\hline Analyte (Drug) & $\begin{array}{c}\text { PGE } \\
\text { Type/Modifier }\end{array}$ & $\begin{array}{l}\text { Analysis } \\
\text { Technique }\end{array}$ & Linear Range (mol/L) & $\operatorname{LoD}(\mathrm{mol} / \mathrm{L})$ & Sample & Ref. \\
\hline $\begin{array}{l}\text { Domperidone } \\
\text { Pantoprazole }\end{array}$ & -/PEBT & AdS-SWV & $\begin{array}{l}1.00 \times 10^{-8}-3.40 \times 10^{-6} \\
4.00 \times 10^{-8}-5.50 \times 10^{-6}\end{array}$ & $\begin{array}{l}4.00 \times 10^{-9} \\
1.20 \times 10^{-8}\end{array}$ & $\begin{array}{l}\text { Synthetic mixture; } \\
\text { human plasma }\end{array}$ & [112] \\
\hline $\begin{array}{l}\text { Ertapenem } \\
\text { Meropenem }\end{array}$ & $-/ \mathrm{PBCG}$ & SWV & $\begin{array}{l}3.00 \times 10^{-7}-7.50 \times 10^{-5} \\
1.00 \times 10^{-6}-6.00 \times 10^{-5}\end{array}$ & $\begin{array}{l}8.00 \times 10^{-8} \\
3.20 \times 10^{-7}\end{array}$ & $\begin{array}{c}\text { Pharmaceutical } \\
\text { vials; rabbit plasma }\end{array}$ & [113] \\
\hline Levofloxacin & 2B/poly(Azure-B) & DPV & $1.00 \times 10^{-6}-1.25 \times 10^{-4}$ & $1.20 \times 10^{-6}$ & Urine; serum & [45] \\
\hline \multirow{3}{*}{ Acetaminophen } & $-/ 2-\mathrm{TBA}$ & DPV & $\begin{array}{l}9.00 \times 10^{-8}-5.00 \times 10^{-7} \\
5.00 \times 10^{-7}-9.46 \times 10^{-6} \\
9.46 \times 10^{-6}-4.23 \times 10^{-4}\end{array}$ & $2.10 \times 10^{-8}$ & $\begin{array}{l}\text { Tap and lake water; } \\
\text { child syrup }\end{array}$ & [59] \\
\hline & $\mathrm{H} / \mathrm{PA} / \mathrm{AgNPs}$ & DPV & $5.00 \times 10^{-8}-8.00 \times 10^{-7}$ & $1.01 \times 10^{-8}$ & \multirow{2}{*}{$\begin{array}{l}\text { Pharmaceutical } \\
\text { tablets and syrup }\end{array}$} & [105] \\
\hline & 2H/SWCNTs/PVP & SWV & $1.00 \times 10^{-6}-5.00 \times 10^{-4}$ & $3.80 \times 10^{-7}$ & & [41] \\
\hline 2-Thiouracil & $\begin{array}{l}\text {-/PA } \\
\text {-/PPy }\end{array}$ & SCV & $\begin{array}{l}1.00 \times 10^{-8}-1.30 \times 10^{-7} \\
1.00 \times 10^{-8}-1.50 \times 10^{-7}\end{array}$ & $\begin{array}{l}1.80 \times 10^{-9} \\
1.60 \times 10^{-9}\end{array}$ & $\begin{array}{c}\text { Pharmaceutical } \\
\text { tablets; serum; urine }\end{array}$ & [109] \\
\hline $\begin{array}{l}\text { 2-Aminobenz- } \\
\text { imidazole }\end{array}$ & HB/MIP (РPy) & DPV & $1.00 \times 10^{-9}-3.00 \times 10^{-2}$ & $1.00 \times 10^{-10}$ & $\begin{array}{l}\text { Chicken, turkey, } \\
\text { beef, and fish meat }\end{array}$ & [114] \\
\hline Benzimidazole & HB/MIP (РPy) & DPV & $3.00 \times 10^{-6}-5.00 \times 10^{-3}$ & $7.00 \times 10^{-7}$ & $\begin{array}{c}\text { Chicken, turkey, } \\
\text { beef, lamb, and fish } \\
\text { meat }\end{array}$ & [115] \\
\hline Methimazole & HB/MIP (РPy) & DPV & $7.00 \times 10^{-6}-6.00 \times 10^{-3}$ & $3.00 \times 10^{-6}$ & $\begin{array}{l}\text { Pharmaceutical } \\
\text { tablets; human } \\
\text { serum }\end{array}$ & [116] \\
\hline Sulfanilamide & -/MIP (PPy) & DPV & $\begin{array}{l}5.00 \times 10^{-8}-1.10 \times 10^{-6} \\
1.10 \times 10^{-6-} 4.80 \times 10^{-5}\end{array}$ & $2.00 \times 10^{-8}$ & $\begin{array}{l}\text { Serum; ground } \\
\text { water }\end{array}$ & [117] \\
\hline Doxicycline & HB/MIP (OPPy) & DPV & $\begin{array}{l}5.00 \times 10^{-5}-5.00 \times 10^{-4} \\
3.00 \times 10^{-3}-7.00 \times 10^{-3}\end{array}$ & $4.35 \times 10^{-5}$ & $\begin{array}{l}\text { Pharmaceutical } \\
\text { capsules }\end{array}$ & [118] \\
\hline Sulfasalazine & 2B/MIP (OPPy) & DPV & $1.00-10.00 \mu \mathrm{g} / \mathrm{mL}$ & $0.26 \mu \mathrm{g} / \mathrm{mL}$ & $\begin{array}{l}\text { Pharmaceutical } \\
\text { tablets }\end{array}$ & [119] \\
\hline Primaquine & $/ \mathrm{C}_{60}-\mathrm{MIP}(\mathrm{TAT})$ & AS-DPV & $2.70 \times 10^{-9}-8.48 \times 10^{-7}$ & $8 \times 10^{-10}$ & $\begin{array}{l}\text { Pharmaceuticals; } \\
\text { plasma; urine }\end{array}$ & [48] \\
\hline $\begin{array}{l}\text { Lamivudine } \\
\text { Zidovudine }\end{array}$ & $\begin{array}{l}\text { /HCS-MIP } \\
\text { (TAT) }\end{array}$ & AS-DPV & $\begin{array}{l}7.26-80.16 \mathrm{ng} / \mathrm{mL} \\
4.76-128.76 \mathrm{ng} / \mathrm{mL}\end{array}$ & $\begin{array}{l}2.23 \mathrm{ng} / \mathrm{mL} \\
1.26 \mathrm{ng} / \mathrm{mL}\end{array}$ & $\begin{array}{l}\text { Pharmaceuticals; } \\
\text { serum }\end{array}$ & [120] \\
\hline Pantoprazole & $\begin{array}{c}\mathrm{HB} / \mathrm{f}- \\
\text { MWCNTs/MIP } \\
\text { (PPy) }\end{array}$ & DPV & $5.00 \times 10^{-6}-7.00 \times 10^{-4}$ & $3.75 \times 10^{-7}$ & $\begin{array}{l}\text { Pharmaceutical } \\
\text { capsules; serum }\end{array}$ & [121] \\
\hline Celecoxib & $\begin{array}{c}-/ \mathrm{f}- \\
\mathrm{MWCNTs} / \mathrm{MIP} \\
\text { (PPy) }\end{array}$ & DPV & $5.00 \times 10^{-9}-2.00 \times 10^{-5}$ & $2.34 \times 10^{-9}$ & $\begin{array}{l}\text { Pharmaceutical } \\
\text { tablets; serum }\end{array}$ & [122] \\
\hline Metoprolol & $\begin{array}{c}-/ \mathrm{f}- \\
\text { MWCNTs/MIP } \\
\text { (PPy) }\end{array}$ & DPV & $6.00 \times 10^{-8}-4.90 \times 10^{-4}$ & $2.88 \times 10^{-9}$ & $\begin{array}{l}\text { Pharmaceutical } \\
\text { tablets; serum }\end{array}$ & [106] \\
\hline Triamterene & $\begin{array}{c}\mathrm{HB} / \mathrm{f}- \\
\text { MWCNTs/MIP } \\
\text { (PPy) }\end{array}$ & DPV & $8.00 \times 10^{-8}-2.65 \times 10^{-4}$ & $3.35 \times 10^{-9}$ & $\begin{array}{l}\text { Pharmaceutical } \\
\text { tablets; serum }\end{array}$ & [107] \\
\hline $\begin{array}{l}\text { Capecitabine } \\
\text { Erlotinib } \mathrm{HCl}\end{array}$ & $\begin{array}{c}\text {-/HF- } \\
\text { MWCNTs/PU }\end{array}$ & DPV & $\begin{array}{l}7.70 \times 10^{-6}-1.42 \times 10^{-4} \\
1.10 \times 10^{-7}-2.35 \times 10^{-5}\end{array}$ & $\begin{array}{l}1.10 \times 10^{-7} \\
2.00 \times 10^{-8}\end{array}$ & Nail; urine & [133] \\
\hline Flutamide & $\begin{array}{l}\text { 2B/HF- } \\
\text { GO/HPB }\end{array}$ & SWV & $1.00 \times 10^{-7}-1.10 \times 10^{-4}$ & $2.90 \times 10^{-8}$ & Plasma & [140] \\
\hline Imatinib & $\begin{array}{l}\text { ET-HB/HF- } \\
\text { PAMAM-rGO }\end{array}$ & DPV & $\begin{array}{l}1.00 \times 10^{-8}-1.00 \times 10^{-5} \\
1.00 \times 10^{-5}-2.00 \times 10^{-4}\end{array}$ & $7.39 \times 10^{-9}$ & Serum; urine & [88] \\
\hline 6-Thioguanine & $\begin{array}{c}\mathrm{HB} / \mathrm{ErGO} / \mathrm{MIP} \\
(\mathrm{PNR})\end{array}$ & AS-DPV & $0.124-78 \mathrm{ng} / \mathrm{mL}$ & $0.02 \mathrm{ng} / \mathrm{mL}$ & $\begin{array}{l}\text { Pharmaceuticals; } \\
\text { serum; urine }\end{array}$ & [49] \\
\hline Epinephrine & $\begin{array}{l}\text { 2B/MIP } \\
\text { (CNT-mer) }\end{array}$ & AS-DPV & $0.09-5.90 \mathrm{ng} / \mathrm{mL}$ & $0.02 \mathrm{ng} / \mathrm{mL}$ & $\begin{array}{l}\text { Pharmaceutical } \\
\text { vials; serum }\end{array}$ & [50] \\
\hline $\begin{array}{c}\text { Folic acid } \\
\text { (vitamin B9) }\end{array}$ & $\begin{array}{c}\text { HB/CNDs/MIP } \\
\text { (PABSA) }\end{array}$ & CS-DPV & $2.20-30.80 \mathrm{ng} / \mathrm{mL}$ & $2.02 \mathrm{ng} / \mathrm{mL}$ & $\begin{array}{l}\text { Pharmaceutical } \\
\text { tablets; urine }\end{array}$ & [123] \\
\hline
\end{tabular}


Table 3. Cont.

\begin{tabular}{|c|c|c|c|c|c|c|}
\hline Analyte (Drug) & $\begin{array}{c}\text { PGE } \\
\text { Type/Modifier }\end{array}$ & $\begin{array}{l}\text { Analysis } \\
\text { Technique }\end{array}$ & Linear Range (mol/L) & LoD (mol/L) & Sample & Ref. \\
\hline $\begin{array}{l}\text { Citaprolam } \\
\text { Fluoxetine } \\
\text { Setraline }\end{array}$ & $\begin{array}{l}\text { H/PVC/PEDOT- } \\
\mathrm{C}_{14}\end{array}$ & ITS-LSV & $1.00 \times 10^{-7}-1.50 \times 10^{-6}$ & $\begin{array}{l}3.50 \times 10^{-8} \\
4.50 \times 10^{-8} \\
2.50 \times 10^{-8}\end{array}$ & Tap and river water & {$[138]$} \\
\hline Cefixime & $\frac{-}{\text { /AuNPs/CTAB }}$ & DPV & $1.00 \times 10^{-8}-3.00 \times 10^{-7}$ & $1.21 \times 10^{-10}$ & $\begin{array}{l}\text { Pharmaceutical } \\
\text { tablets; serum; urine }\end{array}$ & {$[141]$} \\
\hline \multirow{2}{*}{ Ceftizoxime } & $\begin{array}{c}\mathrm{ET}- \\
2 \mathrm{~B} / \mathrm{rGO} / \mathrm{PNCs}\end{array}$ & AdS-DPV & $1.00 \times 10^{-11}-3.00 \times 10^{-8}$ & $1.80 \times 10^{-12}$ & $\begin{array}{l}\text { Pharmaceutical } \\
\text { vials; serum }\end{array}$ & {$[90]$} \\
\hline & /rGO/HÄANPs & AdS-DPV & $\begin{array}{l}1.00 \times 10^{-12}-1.00 \times 10^{-11} \\
1.00 \times 10^{-11}-1.00 \times 10^{-9}\end{array}$ & $3.50 \times 10^{-13}$ & $\begin{array}{l}\text { Pharmaceutical } \\
\text { vials; plasma }\end{array}$ & {$[142]$} \\
\hline Ceftazidime & $\mathrm{HB} / \mathrm{rGO} / \mathrm{HPtNPs}$ & AdS-DPV & $5.00 \times 10^{-13}-1.00 \times 10^{-9}$ & $2.20 \times 10^{-13}$ & $\begin{array}{l}\text { Pharmaceutical } \\
\text { vials; plasma }\end{array}$ & [143] \\
\hline Clozapine & $\begin{array}{c}\text {-/rGO- } \\
\text { X/Nafion } \\
(\mathrm{X}: \mathrm{Au} ; \mathrm{Pd} ; \mathrm{Pt})\end{array}$ & DPV & $5.00 \times 10^{-8}-1.00 \times 10^{-5}$ & $1.60 \times 10^{-9}$ & Serum & {$[135]$} \\
\hline $\begin{array}{l}\text { Pyridoxine } \\
\text { (vitamin B6) }\end{array}$ & $\begin{array}{l}-/ \beta-C D- \\
\text { G/PtNPs }\end{array}$ & DPV & $5.00 \times 10^{-9}-2.05 \times 10^{-7}$ & $1.20 \times 10^{-9}$ & Fruit juice & {$[144]$} \\
\hline Febuxostat & $\begin{array}{l}\text { 2B/AuNPs@f- } \\
\text { CNFs }\end{array}$ & AdS-SWV & $3.00 \times 10^{-8}-7.70 \times 10^{-5}$ & $1.27 \times 10^{-8}$ & $\begin{array}{l}\text { Pharmaceutical } \\
\text { tablets; plasma; } \\
\text { urine }\end{array}$ & {$[17]$} \\
\hline Propranolol & $\mathrm{HB} / \mathrm{TiO}_{2} / \mathrm{MWCNTs}$ & AdS-DPV & $8.50 \times 10^{-8}-6.50 \times 10^{-6}$ & $2.10 \times 10^{-8}$ & $\begin{array}{l}\text { Pharmaceutical } \\
\text { tablets, serum; urine }\end{array}$ & {$[132]$} \\
\hline \multirow{3}{*}{$\begin{array}{l}\text { Riboflavin } \\
\text { (vitamin B2) }\end{array}$} & /AZA/NiHCF & DPV & $4.37 \times 10^{-6}-1.23 \times 10^{-3}$ & $1.45 \times 10^{-6}$ & Vitamin tablets & {$[145]$} \\
\hline & 2B/Chit/Sn & SWV & $1.00 \times 10^{-8}-1.20 \times 10^{-6}$ & $5.56 \times 10^{-9}$ & $\begin{array}{l}\text { Pharmaceuticals; } \\
\text { milk powder }\end{array}$ & [69] \\
\hline & SLSMCNTPGCPE & DPV & $\begin{array}{l}2.00 \times 10^{-7}-8.00 \times 10^{-7} \\
1.00 \times 10^{-6}-5.00 \times 10^{-6}\end{array}$ & $1.16 \times 10^{-8}$ & $\begin{array}{l}\text { B-complex pill; } \\
\text { food supplement }\end{array}$ & {$[72]$} \\
\hline Valproic acid & -/APTES-MNPs & DPV & $1.00-100.00 \mu \mathrm{g} / \mathrm{mL}$ & $0.40 \mu \mathrm{g} / \mathrm{mL}$ & Plasma & [67] \\
\hline \multirow{3}{*}{$\begin{array}{l}\text { Ascorbic acid } \\
\text { (vitamin } C)\end{array}$} & $\begin{array}{l}\text { ET-2B/MIP } \\
\text { (PPy-oPD) }\end{array}$ & SWV & $1.00 \times 10^{-6}-1.00 \times 10^{-3}$ & $2.63 \times 10^{-7}$ & $\begin{array}{l}\text { Pharmaceutical } \\
\text { tablets }\end{array}$ & [89] \\
\hline & $-/ \mathrm{PIG}$ & SWV & $4.00 \times 10^{-5}-4.10 \times 10^{-3}$ & $2.34 \times 10^{-7}$ & - & {$[134]$} \\
\hline & $/ \mathrm{MWCN} \stackrel{-}{\mathrm{TT}} / \mathrm{CeO}_{2} \mathrm{NP}$ & $\mathrm{CV}$ & $4.20 \times 10^{-8}-5.39 \times 10^{-7}$ & $8.00 \times 10^{-9}$ & - & {$[146]$} \\
\hline $\begin{array}{l}\text { L-Ascorbic acid } \\
\text { D-Ascorbic acid }\end{array}$ & $\begin{array}{l}\text {-/MIPANI- } \\
\text { FSA/CDs }\end{array}$ & DPV & $\begin{array}{l}6.00 \times 10^{-9}-1.65 \times 10^{-7} \\
6.00 \times 10^{-9}-1.55 \times 10^{-7}\end{array}$ & $\begin{array}{l}1.00 \times 10^{-12} \\
2.00 \times 10^{-12}\end{array}$ & $\begin{array}{c}\text { Pharmaceutical } \\
\text { tablets; } \\
\text { cerebrospinal fluid; } \\
\text { plasma }\end{array}$ & {$[38]$} \\
\hline $\begin{array}{l}\text { Acetylsalicylic } \\
\text { acid } \\
\text { Ascorbic acid }\end{array}$ & 2B/MWCNTs/SMT & AdS-DPV & $\begin{array}{l}8.05 \times 10^{-7}-1.00 \times 10^{-4} \\
3.19 \times 10^{-7}-6.00 \times 10^{-5}\end{array}$ & $\begin{array}{l}2.41 \times 10^{-7} \\
9.60 \times 10^{-8}\end{array}$ & $\begin{array}{l}\text { Pharmaceutical } \\
\text { tablets }\end{array}$ & {$[136]$} \\
\hline $\begin{array}{l}\text { Acetaminophen } \\
\text { Acetylsalicylic } \\
\text { acid } \\
\text { Ascorbic acid }\end{array}$ & $\frac{\mathrm{ET}-}{\text { 2B/MWCNTs/SEP }}$ & AdS-DPV & $\begin{array}{l}1.28 \times 10^{-8}-1.40 \times 10^{-4} \\
2.13 \times 10^{-8}-1.00 \times 10^{-4} \\
2.01 \times 10^{-8}-1.20 \times 10^{-4}\end{array}$ & $\begin{array}{l}1.80 \times 10^{-8} \\
4.70 \times 10^{-8} \\
4.20 \times 10^{-8}\end{array}$ & $\begin{array}{l}\text { Pharmaceutical } \\
\text { tablets }\end{array}$ & [85] \\
\hline $\begin{array}{l}\text { Acetaminophen } \\
\text { Chlorpheniramine } \\
\text { maleate } \\
\text { Caffeine } \\
\text { Ascorbic acid }\end{array}$ & $\begin{array}{c}\text { 2B/P(L- } \\
\text { met }) / \text { AuNPs }\end{array}$ & DPV & $\begin{array}{l}3.18 \times 10^{-6}-3.00 \times 10^{-3} \\
9.74 \times 10^{-6}-7.14 \times 10^{-4} \\
8.30 \times 10^{-6}-2.00 \times 10^{-3} \\
1.00 \times 10^{-5}-2.00 \times 10^{-3}\end{array}$ & $\begin{array}{l}9.50 \times 10^{-7} \\
2.92 \times 10^{-6} \\
2.50 \times 10^{-9} \\
3.03 \times 10^{-6}\end{array}$ & $\begin{array}{l}\text { Pharmaceutical } \\
\text { powder; serum }\end{array}$ & {$[130]$} \\
\hline Guaifenesin & $\begin{array}{c}\mathrm{HB} / \mathrm{P}(\mathrm{L}- \\
\text { cys }) / \mathrm{AgNPs}\end{array}$ & DPV & $3.00 \times 10^{-8}-3.00 \times 10^{-4}$ & $6.10 \times 10^{-9}$ & $\begin{array}{c}\text { Pharmaceutical } \\
\text { syrup; serum; urine }\end{array}$ & {$[53]$} \\
\hline $\begin{array}{l}\text { Ifosfamide } \\
\text { Etoposide }\end{array}$ & $\begin{array}{l}\text { ET/Au/Pd@ } \\
\text { rGO@P(L-cys) }\end{array}$ & DPV & $\begin{array}{l}1.00 \times 10^{-7}-9.00 \times 10^{-5} \\
1.00 \times 10^{-8}-4.00 \times 10^{-5}\end{array}$ & $\begin{array}{l}9.21 \times 10^{-9} \\
7.18 \times 10^{-10}\end{array}$ & $\begin{array}{l}\text { Pharmaceutical } \\
\text { injections; serum; } \\
\text { urine }\end{array}$ & [87] \\
\hline Vitamin K & $\begin{array}{c}\mathrm{ET} / 2-\mathrm{A}-5- \\
\mathrm{CBP} / \mathrm{AgNPs}\end{array}$ & SWV & $5.00 \times 10^{-8}-7.00 \times 10^{-7}$ & $1.66 \times 10^{-8}$ & Serum & {$[60]$} \\
\hline Mebeverine & $\begin{array}{c}\mathrm{HB} / \mathrm{MIP} \\
(\mathrm{PPy}) / \mathrm{AgNPs}\end{array}$ & DPV & $\begin{array}{l}1.00 \times 10^{-8}-1.00 \times 10^{-6} \\
1.00 \times 10^{-5}-1.00 \times 10^{-3}\end{array}$ & $8.60 \times 10^{-9}$ & $\begin{array}{l}\text { Pharmaceutical } \\
\text { capsules; serum }\end{array}$ & {$[129]$} \\
\hline Levodopa & 2B/GO/DHPA@AgN & JPs DPV & $\begin{array}{l}3.00 \times 10^{-9}-1.00 \times 10^{-7} \\
1.00 \times 10^{-7}-1.00 \times 10^{-5}\end{array}$ & $7.60 \times 10^{-10}$ & $\begin{array}{c}\text { Pharmaceutical } \\
\text { tablets; serum; urine }\end{array}$ & {$[147]$} \\
\hline
\end{tabular}


Table 3. Cont.

\begin{tabular}{|c|c|c|c|c|c|c|}
\hline Analyte (Drug) & $\begin{array}{c}\text { PGE } \\
\text { Type/Modifier }\end{array}$ & $\begin{array}{l}\text { Analysis } \\
\text { Technique }\end{array}$ & Linear Range (mol/L) & LoD (mol/L) & Sample & Ref. \\
\hline Methyldopa & $\mathrm{HB} / \mathrm{GO} / \mathrm{PMoA}$ & DPV & $4.90 \times 10^{-10}-1.00 \times 10^{-7}$ & $1.20 \times 10^{-10}$ & Milk; serum; urine & {$[148]$} \\
\hline Furosemid & $\begin{array}{l}-/ \mathrm{P}[\mathrm{Ni}(\text { salen })] \\
/ \mathrm{Ni}(\mathrm{OH})_{2 /} \mathrm{C} \mathrm{NPs}\end{array}$ & $\mathrm{CV}$ & $2.50 \times 10^{-10}-2.70 \times 10^{-9}$ & $1.40 \times 10^{-10}$ & - & {$[149]$} \\
\hline \multirow{4}{*}{ Insulin } & $\mathrm{ET} / \mathrm{RuO}_{2}-\mathrm{EGO}$ & DPV & $\begin{array}{l}8.00 \times 10^{-10}-2.00 \times 10^{-8} \\
2.00 \times 10^{-8}-1.00 \times 10^{-6}\end{array}$ & $2.40 \times 10^{-11}$ & Plasma; urine & {$[150]$} \\
\hline & $\mathrm{ET} / \mathrm{NiNPs} / \mathrm{MBz}$ & DPV & $2.50 \times 10^{-8}-4.50 \times 10^{-7}$ & $8.30 \times 10^{-9}$ & Serum & {$[20]$} \\
\hline & $\begin{array}{l}\text { ET/NiNPs/Chit/ } \\
\text { MWCNTs } \\
\text { EA/NiONPs/Chit/ } \\
\text { MWCNTs }\end{array}$ & $\mathrm{CV}$ & $\begin{array}{l}1.00 \times 10^{-6}-5.00 \times 10^{-6} \\
5.00 \times 10^{-8}-5.00 \times 10^{-6}\end{array}$ & $\begin{array}{l}4.34 \times 10^{-6} \\
2.60 \times 10^{-8}\end{array}$ & - & {$[68]$} \\
\hline & /CS@Z & DPV & $5.00 \times 10^{-11}-5.00 \times 10^{-8}$ & $1.28 \times 10^{-11}$ & $\begin{array}{l}\text { Pharmaceutical } \\
\text { injections }\end{array}$ & {$[108]$} \\
\hline $\begin{array}{c}\text { Avanafil } \\
\text { Nimodipine }\end{array}$ & $\mathrm{H} / \mathrm{P}(\mathrm{SA}) / \mathrm{NiONPs}$ & SWV & $\begin{array}{l}4.00 \times 10^{-8}-1.00 \times 10^{-6} \\
1.00 \times 10^{-6}-1.50 \times 10^{-5}\end{array}$ & $\begin{array}{l}3.70 \times 10^{-8} \\
3.20 \times 10^{-7}\end{array}$ & Serum & {$[151]$} \\
\hline $\begin{array}{l}\text { Norepinephrine } \\
\text { Uric acid }\end{array}$ & $\begin{array}{c}\text { 2B/DID } \\
\text { (TAT)/f- } \\
\text { MWCNTs/AuNPs }\end{array}$ & AS-DPV & $\begin{array}{l}2.83-44.05 \mathrm{ng} / \mathrm{mL} \\
1.99-44.31 \mathrm{ng} / \mathrm{mL}\end{array}$ & $\begin{array}{l}0.62 \mathrm{ng} / \mathrm{mL} \\
0.43 \mathrm{ng} / \mathrm{mL}\end{array}$ & $\begin{array}{l}\text { Pharmaceuticals; } \\
\text { serum; urine }\end{array}$ & {$[51]$} \\
\hline \multirow{3}{*}{ Tramadol } & $\mathrm{H} / \mathrm{CNT}$ & DPV & $1.00 \times 10^{-6}-2.50 \times 10^{-5}$ & $7.76 \times 10^{-7}$ & Blood serum & [75] \\
\hline & $\begin{array}{c}\text { 2B/f- } \\
\text { MWCNTs/AuNPs }\end{array}$ & DPV & $\begin{array}{l}1.20 \times 10^{-8}-1.00 \times 10^{-7} \\
1.00 \times 10^{-7}-3.00 \times 10^{-6}\end{array}$ & $5.00 \times 10^{-9}$ & $\begin{array}{l}\text { Pharmaceutical } \\
\text { tablets; urine }\end{array}$ & {$[61]$} \\
\hline & -/PPy /CuONPs & SWV & $5.00 \times 10^{-9}-3.80 \times 10^{-4}$ & $1.00 \times 10^{-9}$ & $\begin{array}{l}\text { Pharmaceutical } \\
\text { tablets and vials }\end{array}$ & {$[110]$} \\
\hline $\begin{array}{l}\text { Raloxifene } \\
\text { Tamoxifen }\end{array}$ & $\mathrm{ET} / \mathrm{PPy} / \mathrm{G} / \mathrm{CuO}$ & SWV & $\begin{array}{l}1.00 \times 10^{-8}-5.50 \times 10^{-4} \\
5.00 \times 10^{-7}-4.50 \times 10^{-4}\end{array}$ & $\begin{array}{l}3.00 \times 10^{-9} \\
1.05 \times 10^{-8}\end{array}$ & $\begin{array}{l}\text { Pharmaceutical } \\
\text { tablets; serum }\end{array}$ & {$[86]$} \\
\hline Caffeine & $\begin{array}{c}\text { 2B/MIP } \\
(\mathrm{PPy}) / \text { sol- } \\
\text { gel/AuNPs }\end{array}$ & SWV & $\begin{array}{l}2.00 \times 10^{-9}-5.00 \times 10^{-8} \\
5.00 \times 10^{-8}-1.00 \times 10^{-6}\end{array}$ & $9.00 \times 10^{-10}$ & $\begin{array}{l}\text { Pharmaceutical } \\
\text { tablets; urine; } \\
\text { plasma green tea; } \\
\text { energy and } \\
\text { soda drink }\end{array}$ & [36] \\
\hline Lorazepam & $\begin{array}{c}\text { 2B/MIP } \\
\text { (PPy)/sol- } \\
\text { gel/AuNPs }\end{array}$ & SWV & $\begin{array}{l}2.00 \times 10^{-10}-2.00 \times 10^{-9} \\
2.00 \times 10^{-9}-2.00 \times 10^{-8}\end{array}$ & $9.00 \times 10^{-11}$ & $\begin{array}{l}\text { Pharmaceutical } \\
\text { tablets; plasma; } \\
\text { urine }\end{array}$ & [37] \\
\hline Ranitidine & $\begin{array}{c}\text { 2B/f- } \\
\mathrm{MWCNTs} / \mathrm{MIP} / \mathrm{sol}- \\
\text { gel/AuNPs }\end{array}$ & SWV & $5.00 \times 10^{-8}-2.00 \times 10^{-6}$ & $2.00 \times 10^{-8}$ & Urine & [91] \\
\hline Morphine & $\begin{array}{c}\text { ET/f- } \\
\text { MWCNTs/MIP } \\
\text { (PMT- } \\
\text { MOS)/AuNPs }\end{array}$ & SWV & $8.00 \times 10^{-9}-5.00 \times 10^{-6}$ & $2.90 \times 10^{-9}$ & Plasma; urine & [92] \\
\hline Carbazine & $\begin{array}{c}\text { /f- } \\
\text { MWCNTs/MIP } \\
\text { (PABA)-NSPs }\end{array}$ & AS-DPV & $0.10-50.80 \mathrm{ng} / \mathrm{mL}$ & $0.02 \mathrm{ng} / \mathrm{mL}$ & $\begin{array}{l}\text { Pharmaceuticals; } \\
\text { plasma; urine }\end{array}$ & {$[126]$} \\
\hline $\begin{array}{c}\text { Thiamine } \\
\text { (vitamin B1) }\end{array}$ & $\begin{array}{l}\text { 2B/ANiNPs /f- } \\
\text { MWCNTs/MIP } \\
\text { (PNMGA) }\end{array}$ & AS-DPV & $0.60-19.43 \mathrm{ng} / \mathrm{mL}$ & $0.17 \mathrm{ng} / \mathrm{mL}$ & $\begin{array}{l}\text { Pharmaceutical } \\
\text { tablets; plasma; } \\
\text { urine }\end{array}$ & {$[52]$} \\
\hline $\begin{array}{l}\text { Cyanocobalamine } \\
\text { (vitamin B12) }\end{array}$ & $\begin{array}{c}\text {-/MIP } \\
(\mathrm{MB}-\mathrm{rGO} / \mathrm{f}- \\
\text { MWCNTs/AU) }\end{array}$ & AS-DPV & $0.021-1.44 \mathrm{ng} / \mathrm{mL}$ & $0.0056 \mathrm{ng} / \mathrm{mL}$ & $\begin{array}{l}\text { Pharmaceuticals, } \\
\text { cerebrospinal fluid; } \\
\text { serum; urine }\end{array}$ & {$[125]$} \\
\hline Fexofenadine & $\begin{array}{l}\mathrm{HB} / \mathrm{NDG} / \mathrm{MIP} \\
(\mathrm{PAA}) / \text { sol-gel }\end{array}$ & DPV & $5.00 \times 10^{-10}-7.80 \times 10^{-6}$ & $1.50 \times 10^{-10}$ & $\begin{array}{c}\text { Pharmaceutical } \\
\text { tablets, blood; urine }\end{array}$ & [127] \\
\hline Isoniazid & $\begin{array}{c}\text { ET/CNQDs/Cu- } \\
\text { MOF }\end{array}$ & DPV & $1.00 \times 10^{-7}-6.5 \times 10^{-5}$ & $6.75 \times 10^{-8}$ & $\begin{array}{l}\text { Pharmaceutical } \\
\text { tablets; serum }\end{array}$ & [62] \\
\hline Setraline & $\begin{array}{l}\mathrm{HB} / \mathrm{SNDG} / \mathrm{Cu}- \\
\mathrm{MOF}\end{array}$ & DPV & $5.00 \times 10^{-8}-2.67 \times 10^{-6}$ & $3.80 \times 10^{-8}$ & $\begin{array}{l}\text { Pharmaceutical } \\
\text { tablets; serum }\end{array}$ & [63] \\
\hline Paroxetine & $\mathrm{H} / \mathrm{PWA} / \mathrm{rGO}$ & DPV & $8.00 \times 10^{-9}-1.00 \times 10^{-6}$ & $9.00 \times 10^{-10}$ & $\begin{array}{l}\text { Pharmaceutical } \\
\text { tablets; serum; urine }\end{array}$ & [64] \\
\hline $\begin{array}{l}\text { Sumatripan } \\
\text { Paroxetine }\end{array}$ & $\begin{array}{l}\mathrm{H} / \mathrm{MIP}(\mathrm{PMA}) / \mathrm{sol}- \\
\text { gel/PWA/rGO }\end{array}$ & AdS-DPV & $\begin{array}{l}2.00 \times 10^{-8}-3.00 \times 10^{-6} \\
5.00 \times 10^{-9}-2.20 \times 10^{-6}\end{array}$ & $\begin{array}{l}4.00 \times 10^{-9} \\
7.00 \times 10^{-10}\end{array}$ & $\begin{array}{l}\text { Pharmaceutical } \\
\text { tablets; serum; urine }\end{array}$ & [65] \\
\hline
\end{tabular}


Table 3. Cont.

\begin{tabular}{|c|c|c|c|c|c|c|}
\hline Analyte (Drug) & $\begin{array}{c}\text { PGE } \\
\text { Type/Modifier }\end{array}$ & $\begin{array}{l}\text { Analysis } \\
\text { Technique }\end{array}$ & Linear Range (mol/L) & LoD (mol/L) & Sample & Ref. \\
\hline Donepezil & $\begin{array}{c}\text { /PSBT/N- } \\
\text { CNDs/CoNPs }\end{array}$ & DPV & $1.50 \times 10^{-9}-4.00 \times 10^{-4}$ & $5.00 \times 10^{-10}$ & $\begin{array}{l}\text { Pharmaceutical } \\
\text { tablets; rabbit } \\
\text { plasma }\end{array}$ & {$[131]$} \\
\hline \multirow{2}{*}{$\begin{array}{l}\text { 6-mercapto- } \\
\text { purine }\end{array}$} & $\begin{array}{c}\text { 2B/IL/N- } \\
\text { HCNS@Pd- } \\
\text { MIP (N-AAsp) }\end{array}$ & AS-DPV & $0.80-70.75 \mathrm{ng} / \mathrm{mL}$ & $0.11-0.22 \mathrm{ng} / \mathrm{ml}$ & $\begin{array}{c}\text { Water; } \\
\text { L pharmaceuticals; } \\
\text { plasma; urine }\end{array}$ & {$[128]$} \\
\hline & $\begin{array}{c}\text {-/MIP } \\
(\mathrm{PPy}) / \text { sol- } \\
\text { gel/ZnO@GQDs }\end{array}$ & DPV & $\begin{array}{l}1.00 \times 10^{-8}-5.00 \times 10^{-5} \\
5.00 \times 10^{-5}-7.00 \times 10^{-4}\end{array}$ & $5.72 \times 10^{-9}$ & $\begin{array}{l}\text { Pharmaceutical } \\
\text { tablets; serum; urine }\end{array}$ & {$[124]$} \\
\hline
\end{tabular}

ET = electrochemically treated; NPs = nanoparticles; MPs = microparticles; MWCNTs = multi-wall carbon nanotubes; TyB = Trypan blue; $\mathrm{PXO}=$ poly(xylenol orange); $\mathrm{PEBT}=$ poly $($ eriochrome black $\mathrm{T})$; $\mathrm{PBCG}=$ poly(bromocresol green); 2-TBA = 2-thiobarbituric acid; PA = polyaniline; SWCNTs = single-walled carbon nanotubes; PVP = poly(vinylpyrrolidone); $\mathrm{PP}=$ polypyrrole; $\mathrm{MIP}=$ molecularly imprinted polymer; OPPy = overoxidized polypyrrole; $\mathrm{C}_{60^{-}}=$fullerene monoaduct; $\mathrm{TAT}=$ 2,4,6-trisacrylamido-1,3,5-triazine HCS = hollow core shell; f-MWCNTs = functionalized MWCNTs; HF = hollow fiber; GO = graphene oxide; $\mathrm{PU}=$ polyurethane; $\mathrm{HPB}=$ hyper-branched polyglycerol; PAMAM = ethylenediamine core poly (amidoamine) dendrimer; rGO = reduced graphene oxide; ErGO = electrochemically rGO; PNR = poly neutral red; $\mathrm{CNT}-\mathrm{mer}=\mathrm{f}-\mathrm{MWCNT}$ monomer; $\mathrm{CNDs}=$ carbon nanodots; $\mathrm{PABSA}=$ poly $(\mathrm{p}$-aminobenzene sulphonic acid); PVC/PEDOT- $\mathrm{C}_{14}=$ polyvinyl chloride/C14 alkyl group-derivatized poly(3,4-ethylenedioxythiophene) $\mathrm{CTAB}=$ cetyltrimethyl ammonium bromide; PNCs = Preyssler nanocapsules; HAuNPs and HPtNPs = hollow $\mathrm{Au} / \mathrm{Pt} \mathrm{NPs} ; \quad \beta-\mathrm{CD}=\beta$-cyclodextrine; $\mathrm{G}=$ graphene; AuNPs@f-CNFs = AuNP-decorated functionalized carbon nanofibers; AZA/NiHCF = Azure A/nickel hexacianoferrate; Chit $/ \mathrm{Sn}=\mathrm{Chitosan} / \mathrm{tin}$ SLSMCNTPGCPE = sodium lauryl sulphate-modified carbon nanotube pencil graphite composite paste electrode; APTES-MNPs = 3-aminopropyletriethoxy silane-coated magnetic NPs; oPD = o-phenylenediamine; PIG = graphene-iron oxide-polyvinyl alcohol; MIPANI-FSA/CDs = molecularly imprinted polyanilineferrocenesulfonic acid/carbon dots; SMT = nano-smectite clay; SEP = Sepiolite clay; P $(\mathrm{L}-\mathrm{met})=$ poly $(\mathrm{L}-$ methionine $)$ P $(\mathrm{L}-c y s)=$ poly $($ L-cysteine $) ; 2-\mathrm{A}-5-\mathrm{CBP}=2$-amino-5-chloro benzophenone; DHPA = Dawson heteropolyacid; $\mathrm{PMoA}$ = phosphomolybdic acid; $\mathrm{P}\left[\mathrm{Ni}(\right.$ salen) $]=$ poly $\left(\mathrm{N}, \mathrm{N}^{\prime}\right.$-bis(salicylidene)ethylenediaminonickel(II) complex); $\mathrm{EGO}=$ exfoliated graphene oxide; $\mathrm{MBz}=$ methylbenzoate; $\mathrm{CS} @ \mathrm{Z}=$ cerium (III) salen encapsulated into dealuminated zeolite $\mathrm{Y} ; \mathrm{P}(\mathrm{SA})=$ poly (sulfanilamide); DID = double-imprinted dendrimer; PMTMOS = poly (methyltrimethoxysilane); PABA = poly (N-acryloylamino butyric acid); NSPs = nanospherical particles; ANiNPs = acryloylated nickel nanoparticles; PNMGA = poly(N-methacryloylglutamic acid); MB = methyl blue $\mathrm{AU}=$ acryloylurea; NDG = nitrogen-doped graphene; PAA = poly(acrylamide); CNQDs = carbon nitride quantum dots; Cu-MOF = copper-based metal organic framework; SNDG = sulfur and nitrogen co-doped graphene; PWA = phosphotungstic acid; PMA = poly (methacrylic acid); PSBT = poly solochrome black T; N-CNDs = nitrogendoped CNDs; IL/N-HCNS@Pd-MIP (N-AAsp) = N-acryloyl derivative of aspartic acid-based MIP of N-doped hollow carbon nanosphere - palladium nanocomposite grafted on ionic liquid (1-butyl-3-methylimidazolium tetrafluorobarate); ZnO@GQDs = ZnO-graphene-capped quantum dots; SCV = staircase voltammetry; AS-DPV: anodic stripping differential pulse voltammetry; CS-DPV = cathodic stripping differential pulse voltammetry; ITS-LSV = ion-transfer stripping linear sweep voltammetry.

As was already stated, modified PGEs have superior electrochemical performance characteristics when compared with the bare ones. For example, propranolol exhibited a small oxidation peak for the unmodified PGE, while for the MWCNT/PGE and $\mathrm{TiO}_{2} / \mathrm{MWCNT} / \mathrm{PGE}$, the peak currents were higher and the potentials shifted due to the presence of carbon nanotubes and $\mathrm{TiO}_{2}$ nanoparticles, which enhanced the electroactive surface area and reduced the electron transfer resistance [132]. A similar behavior was observed for paroxetine, whose weak oxidation signal for the bare PGE increased with the rGO/PGE and furthermore for the PWA/rGO/PGE, along with a decrease in the peak potential [64]. Despite the fact that clozapine was determined in the same linear range by using a PGE either bare or modified with rGO, rGO-Au, rGO-Pd, or rGO-Pt nanocomposites, the LoD of the DPV method varied as follows: $1.04 \times 10^{-8}>8.60 \times 10^{-9}>4.50 \times 10^{-9}>3.60 \times 10^{-9}>1.60 \times 10^{-9} \mathrm{~mol} / \mathrm{L}$ [135]. The acetaminophen (AAP) oxidation peak was small for the bare and PA-modified PGE, whereas for the AgNPs/PA/PGE it was well-defined, with its current significantly increased and its potential shifted toward lower values due to the catalytic effect of AgNPs/PA on the electrode surface [105]. Similarly, tramadol did not show a well-defined peak for the PGE, but after the electrode modification with f-MWCNTs, a clear anodic signal was observed, while further modification with AgNPs resulted in an increased oxidation peak that appeared at lower values due to the enhanced surface area and the synergistic effects of the 
two modifiers' electrocatalytic properties [61]. The electro-oxidation response of guaifenesin for the bare PGE and PGE modified with AgNPs/P(L-cys) was evaluated by CV and DPV. For the unmodified PGE, the guaifenesin alcohol group gave a weak oxidation signal whose peak current increased, and its potential shifted in the negative direction after the electrode surface covering by $\mathrm{P}(\mathrm{L}$-cys $)$ and by $\mathrm{P}(\mathrm{L}$-cys $)$ / AgNPs due to the analyte adsorption through the hydrogen bonding interaction of guaifenesin $-\mathrm{OH}$ groups and the $\mathrm{P}(\mathrm{L}-\mathrm{cys})$ film and the AgNPs' electrocatalytic effect on the resulting current, respectively [53]. The examples could be continued, but generally, it can be concluded that PGE modification generates higher peak currents, yielding more sensitive determinations, and in some cases, a potential shift toward lower values was observed, indicating that the electrode process took place easier.

It is worth mentioning that most voltametric methods developed for pharmaceutical quantification using bare or modified PGEs are based on the analyte oxidation processes (Figure 2a). However, there were a few examples where the reduction signals (Figure 2b) of the species of interest were exploited. Thus, although niclosamide was determined based on the oxidation signal resulting after the electrochemical reduction of its nitro group, it was emphasized that the PGE could also be used successfully to investigate cathodic processes due to the fact that it had a wide potential window (in the mentioned study, from -0.80 to $0.80 \mathrm{~V}$ ) [21]. Additionally, chloramphenicol was quantified for the PGE by SWV considering the reduction peak of its nitro group and by LSV based on the oxidation signal of the $-\mathrm{NHOH}$ moiety contained in its reduction product. The two methods had similar performance characteristics (Table 1) [29]. In the case of a mixture of epinephrine (EP) and norepinephrine (NP), the analytes' oxidation signals for the ET-PGE could not be employed for their simultaneous determination, but the cathodic peaks at $0.15 \mathrm{~V}$ and about $-0.25 \mathrm{~V}$, attributed to the reduction of the EP and NP oxidation products, enabled the direct detection of NP by exploiting the signal from $+0.15 \mathrm{~V}$ and the assessment of the sum of the two catecholamines, considering the current of the signal situated at negative potential values [96,97].

The nitroso group in the lomustine structure [103] and the nitro moiety in the metronidazole molecule [104] allowed the possibility of determining voltametrically these compounds by using their cathodic signals obtained for the mercury and bismuth film-modified PGEs, respectively (Table 3). Flutamide was quantified for the GO/HPB/HF-PGE based on the $-\mathrm{NO}_{2}$ reduction peak [140] (Table 3). CAdS-SWV recordings for a PGE in the range from -0.20 to $-1.20 \mathrm{~V}$ were exploited to determine either individually or simultaneously the flutamide $\left(\mathrm{E}_{\mathrm{p}} \approx-0.50 \mathrm{~V}\right)$ and irinotecam $\left(\mathrm{E}_{\mathrm{p}} \approx-1.00 \mathrm{~V}\right)$ (Table 1) [22] or the nanoacetylene black-modified PGE for the singular or concomitant quantification of flutamide $\left(\mathrm{E}_{\mathrm{p}} \approx-0.80 \mathrm{~V}\right)$ and cyproterone $\left(\mathrm{E}_{\mathrm{p}} \approx-1.20 \mathrm{~V}\right)$ (Table 3) [66].

The reduction signal of vitamin B12 $\left(\mathrm{E}_{\mathrm{p}} \sim-0.80 \mathrm{~V}\right)$ and of vitamin $\mathrm{B} 9\left(\mathrm{E}_{\mathrm{p}} \sim-0.60 \mathrm{~V}\right)$ constituted the basis of their determination by DPV for the bare PGE (Table 1) [55] and by CS-DPV for the CNDs/MIP(PABSA)/PGE [123], respectively, while Sangeetha and Narayanan [144] developed a PGE modified with two mediators, Azure A and nickel hexacianoferrate, with electrocatalytic activity for the oxidation of the antioxidant butylated hydroxyanisole and the reduction of vitamin B2, respectively. Vitamin K1 quantification by SWV for the 2-A-5-CBP/AgNPs/ET-PGE [60] and celecoxib by DPV for the $\mathrm{fMWCNT} / \mathrm{MIP} / \mathrm{PGE}$ [122] were also based on their reduction signals (Table 3).

For the APTES-MNP-modified PGE, valproic acid (Table 3) presented reduction peaks at -0.20 and $-0.80 \mathrm{~V}$, with one situated at more cathodic potentials being exploited for the drug's quantitative determination by DPV [67].

Other molecules were indirectly detected based on the linear decrease of the voltametric signal of a redox probe, such as $\left[\mathrm{Fe}(\mathrm{CN})_{6}\right]^{4-}$ for lamivudine and zidovudine $[120,152]$ and $\left[\mathrm{Ru}\left(\mathrm{NH}_{3}\right)_{6}\right]^{2+}$ for thiamine [52], in the presence of increasing analyte concentrations. 


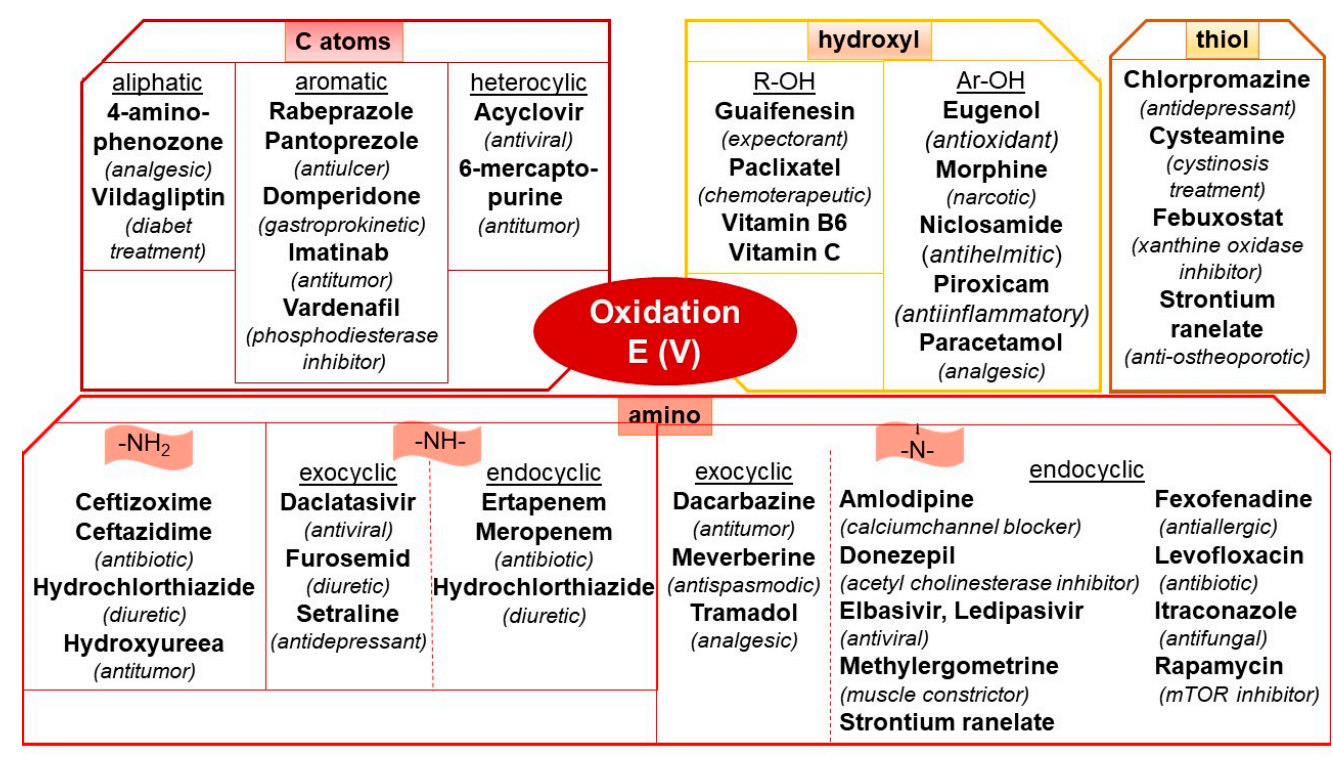

(a)

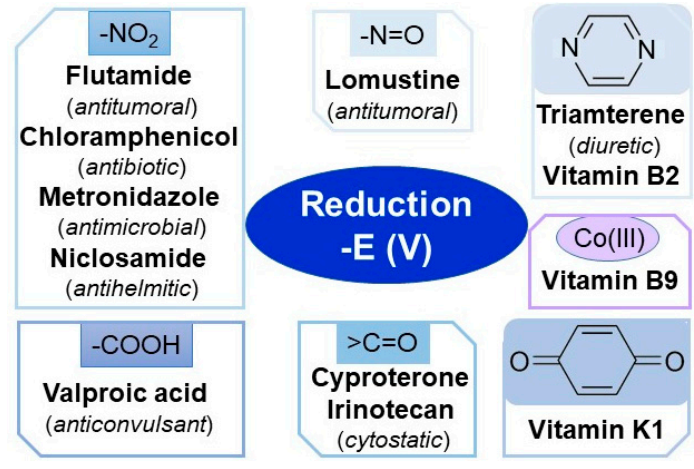

(b)

Figure 2. Examples of electroactive sites generating the (a) anodic and (b) cathodic signals employed for the voltametric analysis of the corresponding pharmaceuticals.

\subsection{Simultaneous Voltametric Determination of Drugs Using PGEs}

Most voltametric methods using PGEs reported in the literature were applied to the determination of a single drug from various matrices. However, there are papers presenting the simultaneous quantification of two, three, or even four pharmaceuticals.

Using a bare PGE, vitamin B1 and vitamin B6 were determined by DPV with one in the presence of the other by changing the supporting electrolyte, due to the fact that vitamin B1 is electroactive only in a $\mathrm{NaOH}$ solution (Table 1) [33], while the oxidation peak separation of more than $1.10 \mathrm{~V}$ obtained in $\mathrm{BRB}$ pH 5.00 enabled the precise and accurate concomitant SWV quantification of vitamin B2 and B6 [79]. EP and NP were determined simultaneously using CSWV [96] and SWV [97] for the ET-PGE.

Ascorbic acid (AA) and acetaminophen (AAP) were determined in their mixture solution, applying AdS-DPV on the SMT/MWCNT/PGE by keeping constant the concentration of one analyte and increasing linearly the concentration of the other and vice versa, as well as by enhancing the concentrations of both drugs at the same time. No differences were observed between the determinations of AAP and AA alone or simultaneously [136]. Similarly, by employing DPV, it was possible to quantify capecitabine and erlotinib hydrochloride for the MWCNT/PU/HF-PGE [133] and ifosfamide and itoposide for the Au/Pd@rGO@P(L-cys)/PGE [87]. CAdS-SWV detection of flutamide and irinotecan (Table 1) [22] or flutamide and cyproterone (Table 3) [66] in mixtures were reported for the bare or nanoacetylene black-modified PGEs, respectively. Using AdS-DPV for a selective $\mathrm{MIP} /$ sol-gel/PWA/rGO/PGE, the sumatripan and paroxetine levels were determined 
with good recoveries from the urine and serum samples by applying the standard addition method [65]. The slopes of the calibration curves for the SWV determination of raloxifene and tamoxifen levels for the PPy/G/CuO/ET-PGE in a mixture were similar to those obtained for each one alone, demonstrating that the method is adequate for simultaneous drug quantification [86]. On the same basis, the DID (TAT)/f-MWCNT/AuNPs/PGE was applied to the AS-DPV determination of both NP and uric acid (UA) in aqueous solutions and in pharmaceutical and biological (blood and urine) samples [51].

An interesting approach for AS-DPV detection of the electro-inactive anti-HIV drugs lamivudine (3TC) and zidovudine (AZT) employed a double-layered one-by-one imprinted (the outer layer imprinted with AZT and the inner layer with 3TC) hollow core shells@PGE. The method exploited the decrease in the $\left[\mathrm{Fe}(\mathrm{CN})_{6}\right]^{4-}$ probe oxidation signal in the presence of increasing concentrations of one of the anaytes (e.g., AZT) when the cavities of the corresponding MIP (the inner layer) were saturated with the other target compound (3TC). The obtained linear ranges and detection limits were $0.50-8.12 \mathrm{ng} / \mathrm{mL}$ and $0.13 \mathrm{ng} / \mathrm{mL}$ for AZT and 3.58-48.98 ng/L and $0.94 \mathrm{ng} / \mathrm{mL}$ for 3CT, respectively, in the blood serum and $0.82-10.55 \mathrm{ng} / \mathrm{mL}$ and $0.20 \mathrm{ng} / \mathrm{mL}$ for AZT and 3.77-56.64 $\mathrm{ng} / \mathrm{L}$ and $0.99 \mathrm{ng} / \mathrm{mL}$ for 3CT, respectively, in the pharmaceutical samples [152].

By applying DPV to the ET-PGE, well-separated and sharp peaks were obtained for acetylsalicylic acid (ASA), NP, and UA. When the concentration of an analyte was increased while maintaining the concentrations of the other two constant, as well as when the concentrations of all three species were gradually enhanced, the peak currents of all compounds varied linearly, and the slopes of the calibration graphs of each analyte did not change significantly, thus enabling their quantitative simultaneous determination [84].

The AA, AAP, and ASA from a ternary mixture were determined with one in the presence of the other two by AdS-DPV for the SEP/MWCNTs/ET-PGE either by varying the concentration of the investigated analyte at constant concentrations of the other two species or by increasing all their concentrations [85], while AA, AAP, chlorpheniramine maleate, and caffeine were simultaneously determined by DPV for the AuNPs/P(L-met)/PGE [130].

\subsection{Pharmacokinetic Studies Performed by Voltammetric Techniques at PGEs}

AS-SWV for the PGE in the presence of $\mathrm{Co}^{2+}$ was applied to the pharmacokinetic study of pantoprazole sodium in rabbit plasma after drug administration. The obtained pharmacokinetic parameters were in good agreement with those obtained by a reported method, thus indicating the reliability of the voltametric method [82]. SWV for the POX/PGE was applied to study the possible pharmacokinetic interaction between vardenafyl hydrochloride (VAR), a drug used to control erectile dysfunctions, and the antiviral drug daclatasivir hydrochloride (DAC), prescribed to patients with Hepatitis C, when they were co-administrated. Investigations performed on spiked rabbit plasma evidenced a higher VAR plasmatic concentration when it was administrated simultaneously with DAC [46]. The possible pharmacokinetic interaction between avanafil used to treat erectile dysfunction and the antihypertensive nimodipine, which are co-administered for elderly patients, was investigated in the plasma of healthy volunteer men using SWV for a NiONPs/P(SA)/PGE [151]. SWV for a PBCG/PGE was successfully applied to pharmacokinetic studies after individual administration of meropenem and ertapenem by monitoring the time-dependent antibiotics levels in healthy rabbit plasma [113]. The pharmacokinetic parameters of donepezil $\mathrm{HCl}$ in rabbits' plasma after drug consumption were determined by CV for a PSBT/N-CNDs/CoNPs/PGE by applying a non-compartmental statistics model using moment analysis [131].

\section{PGEs as Electrochemical Detectors in Separation Techniques}

Real samples as biological ones and wastewaters resulted in the pharmaceutical industry possibly having very complex matrices, and some analytes may exist at concentrations below the detection limit of the analytical method. Therefore, the direct determination of certain species is impossible due to the presence of interfering compounds and the low 
analyte(s) content(s). Separation techniques are recognized as having the ability to isolate the analyte from the matrix sample or concentrate it in another medium with a simple chemical composition. Coupling an electrochemical method using a PGE as a working electrode with separation and extraction techniques enhances both the selectivity and the sensitivity of the quantitative determination of interest species in complex samples.

Liquid three-phase microextraction (LPME) consists of analyte transfer from the aqueous donor phase to a thin layer of a proper organic solvent immobilized within a porous membrane, followed by the re-extraction of it into a smaller volume of an aqueous acceptor phase. A polytetrafluoroethylene (PTFE) membrane impregnated with propyl benzoate was used to transport buprenorphine from the sample (aqueous phase) to the acceptor phase, and a MWCNTs/ET-PGE was used to study the electrochemical behavior of this pharmaceutical and to quantify it by DPV [137]. A similar extraction-electrochemical set-up was described by the same authors for insulin determination. The supported liquid membrane of a PTFE film containing sodium dodecyl sulfate was prepared to transfer the analyte from the sample into a smaller volume of aqueous receiving phase, where insulin was determined at an ultra-trace level by DPV at a $\mathrm{RuO}_{2}$-EGO/ET-PGE [150] (Table 3). LPME-DPV was applied to the detection of these two drugs in human plasma and urine.

Solid phase microextraction (SPME) involves the use of fibers which are not very selective for samples with complex matrices, such as the clinical ones. To overcome this inconvenience, the fiber can be supported with a hollow fiber (HF) membrane in the electromembrane extraction (EME) technique. In EME, the charged analyte is extracted from the sample and into a supported liquid membrane (SLM), which comprises an organic solvent located in the wall pores of the HF, and then the analyte passes into an acceptor phase located inside the lumen of the porous fiber. Pencil lead spin-coated with an rGO layer (rGO/PGE) was used as cathode of the EME unit, and it was introduced in the acceptor phase, existing inside the lumen of the porous HF, having the walls impregnated with 2-nitrophenyl octyl ether as organic extraction solvent (acting as SLM). A Pt wire (anode) was immersed into the stirred sample solution (donor phase). By applying an electrical field between the cathode and the anode, tramadol was extracted from the sample through the SLM, into the receiving solution, and then adsorbed on the rGO/PGE, which acted as a solid adsorbent for SPME. Subsequently, the rGO/PGE with the adsorbed drug was introduced into an electrochemical cell containing only the electrolyte, and linear sweep voltammograms were registered in order to quantify the analgesic. The peak current was proportional to the tramadol concentration in the ranges of $0.01-0.50$ and $0.50-50 \mu \mathrm{g} / \mathrm{mL}$, with an LoD of $0.003 \mu \mathrm{g} / \mathrm{mL}$ [153]. The anticancer drug imatinib was determined from biological samples applying a similar principle, based on microextraction using PAMAM dendrimer-assisted rGO as an adsorbent (immobilized on the ET-PGE and in the HF walls) and DPV detection using the HF-modified ET-PGE (Table 3) [88].

Despite the advantages of the above-mentioned procedures, the most powerful separation technique remains chromatography. The leading analytical methods for drug determination at very low concentrations in various samples are gas and liquid chromatography coupled with mass spectrometric detection (GS/MS/MS; LC/MS/MS). However, chromatographic methods involving a MS detector are very expensive compared with those using spectrometric or electrochemical detection, which are significantly more affordable. Analytes possessing redox properties can be detected online using electrochemical sensors with higher sensitivity and selectivity than those provided by UV-VIS spectrometric detectors. A high-performance liquid chromatographic method coupled with an electrochemical detector based on micro carbon pencil lead as a working electrode was developed for the low-cost and sensitive screening of nine antipsychotic drugs in human plasma [154]. A Pt wire auxiliary electrode and a miniaturized $\mathrm{Ag} / \mathrm{AgCl}$ reference electrode were placed in close proximity to the pencil lead, which was inserted into the HPLC capillary outlet, and the drugs separated by a reverse-phase mechanism were detected, applying a potential of $0.90 \mathrm{~V}$ to the in-capillary working electrode. The calibration plots were linear in the range from the corresponding LoD (situated between $1.00 \times 10^{-9} \mathrm{~mol} / \mathrm{L}$ and $7.90 \times 10^{-9} \mathrm{~mol} / \mathrm{L}$ 
for olanzapine and chlorpromazine, respectively) up to $5.00 \times 10^{-7} \mathrm{~mol} / \mathrm{L}$, proving to be very good inter-electrode reproducibility.

\section{Development of PGE-Reliant Analytical Procedures Aided by Laborious Calculations}

Nowadays, a significant number of papers reporting electrochemical analysis are complemented by complex calculations run by specialized software (one can find the appropriate details in the articles cited in this section). This is because computational chemistry, which is grounded in quantum mechanics, is able to predict the most stable structures to be found in complex, well-specified chemical systems. Meanwhile, the science called chemometrics developed powerful mathematical tools suitable for the optimization of rather complex (electro)analytical procedures or other types of chemical processes. For example, in the case of the cefdinir SWV determination for a bare PGE, a chemometric strategy was employed to select the optimum values of the supporting electrolyte $\mathrm{pH}$, and the frequency and amplitude of the square wave [23].

Although the problems solved by computational chemistry and chemometrics are different, one can also find situations where they contribute symbiotically to the development of robust procedures of pharmaceutical electroanalysis [121], as discussed at the end of this section. Subsequently, this section states briefly the problems solved in the reviewed articles either by chemometrics $[23,88,106,107,121,122,129,142,143,153]$ or computational chemistry $[48-50,86,106,107,114,115,117,121,122]$.

Primarily to select the optimum monomer used for MIP preparations, quantum mechanics calculi have been performed $[106,107,114,117,121,122]$ in order to maximize the energy difference between the monomer and template on one side and their association on the other side in a pre-polimerization solution, with the rationale that the bigger this difference is, the more stable the recognition sites formed in the MIP during electropolymerization should be. Pyrrole [106,107,114,115,117,121,122] was selected and employed in the subsequent works as the optimum monomer among the tested ones: furan $[106,107,114,117,121,122]$, thiophene $[106,107,114,117,121,122]$, methylthiophene $[114,117,121,122]$, and methylpyrrole $[106,107,117,121,122]$. Where only pyrrole was considered a monomer [115], the stoichiometry of the most stable association between benzimidazole and pyrrole was found to be 1:2. Most articles of this type also display the geometrical conformations of the most stable associations (called "complexes") found by the employed calculus $[106,107,114,115,117,121]$.

In the design of more complex materials also containing polymers in their compositions, the optimum monomer was selected among a few options by ab initio calculations, as was the case for the modified PGEs employed in the determinations of primaquine [48], 6thioguanine [49], and epinephrine [50], respectively. Finally, Fouladgar et al. [86] performed density functional theory calculations with van der Waals correction in order to explain the behavior difference between tamoxifen and raloxifene on a CuO/G/PPy/ET-PGE. The results of the calculi explain the strong adsorption on $\mathrm{CuO}$ of raloxifene, which was found experimentally.

Depending on the nature of the structured materials engineered to cover the PGE surface, the number of experimental variates that influence the sensors' performance during the determinations can be numerous. Faced with the need to optimize many experimental parameters, multifactorial optimizations of the developed analytical procedures have been performed either by classical methods, such as Analysis of Variance (ANOVA) or the Response Surface (RS) methodology [88,105-107,129,142,143,153], or by more advanced chemometric tools, such as Artificial Neural Networks (ANNs) [121,122] or Genetic Algorithms (GAs) [121,122]. As input for the employed optimization methods, data acquired in one or two sets of developed experiments have been used, with the aim of limiting the number of determinations according to the available experimental designs of the following types: Plackett-Burman (PB) [106,107,121,122,129], central composite (CCD) $[104,106,121,122,129,142,143]$, Box-Behnken [153], or Taguchi L25 orthogonal [88]. 
For example, the conditions (e.g., $\mathrm{pH}, \mathrm{Bi}^{3+}$ concentration, deposition time, and potential) for the preparation of the Bi film-modified PGE used for metronidazole voltammetric investigation were optimized by applying a circumscribed CCD [104], while a full factorial design with three factors at three levels was used to establish the optimum $\mathrm{pH}$, accumulation time, and accumulation potential in the fabrication of a AgNPs/PA/PGE for the analysis of acetaminophen [105].

As a relevant example of optimization significance in achieving good performance characteristics of complex electrochemical sensors, the procedure followed for the MIP (PPy)/f-MWCNTs/PGE [121] is briefly described below. This example was chosen because the optimization of the MIP/f-MWCNTs/PGE fabrication was compared during the entire process with the one for a non-imprinted PGE sensor (NIP/f-MWCNTs/PGE) obtained in a similar fashion to the MIP/f-MWCNTs/PGE but without a template in the electropolymerization solution and used in parallel sets of analytical measurements. After pyrrole selection as the monomer for MIP generation by ab initio calculations, eight parameters ( $\mathrm{pH}$ of the buffer solution, $\mathrm{CV}$ scan number and rate in the polymerization step, monomer and template concentrations in the prepolymerization mixture, nanotube concentration in f-MWCNTs-coating step, and the uptake time and stirring rate after MIP preparation) were considered in 12 randomized runs developed in agreement with a PB factorial design due to the ability of this optimization method to identify the main factors affecting the measurements in relatively few experiments. During each run, the current peak heights were the responses obtained at two levels of factors. Data analysis of the obtained results identified as significant, in decreasing order, the following three factors: monomer concentration in the electropolymerization solution, buffer $\mathrm{pH}$, and uptake stirring rate. Due to the strong interactions between those three factors, a CCD was subsequently employed. It consisted of a full factorial design, six axial points, six center points, and eight cube points in order to fit the quadratic polynomials. In addition to the classical mathematical treatment of the data, by modeling the response surface, the two responses of the MIPand NIP/f-MWCNTs/PGEs were fed to the two neurons from the first layer of an artificial neural network or in a genetic algorithm that simulated the behavior of simple biological systems and was programmed in MATLAB. The optimum conditions achieved by the two modeling methods of the measurement system (i.e., ANN and GA) were as follows: $1.00 \times 10^{-4} \mathrm{~mol} / \mathrm{L}$ template (pantoprazole), $50 \mathrm{CV}$ cycles, $4.30 \times 10^{-3} \mathrm{~mol} / \mathrm{L}$ monomer (pyrrole), and $0.10 \mathrm{~mol} / \mathrm{L}$ supporting electrolyte $\left(\mathrm{NaClO}_{4} \cdot \mathrm{H}_{2} \mathrm{O}\right)$ for $\mathrm{MIP}$ and the same conditions but without the template for NIP fabrication. The complex optimization procedure performed resulted in a maximum response ratio of 17.4 between the currents recorded by the MIP- and NIP/f-MWCNTs/PGE sensors for the same samples and, consequently, good performance characteristics for the pantoprazole determinations (Table 3).

\section{PGE Surface Characterization}

To confirm the change of the PGE surface during the various steps performed for its modification, several analytical techniques (electrochemical, spectroscopic, and microscopic) were employed. Usually, the selected parameters (e.g., peak potentials and currents, charge transfer resistances, wavelengths, and wavenumbers) of the modifier(s) of the bare and final or intermediate modified PGE were compared.

\subsection{PGE Surface Characterization by Electrochemical Techniques}

The electrochemical characterization of bare and modified electrodes was usually performed by CV [87,88,117,124,125,129] and electrochemical impedance spectroscopy (EIS) using $\left[\mathrm{Fe}(\mathrm{CN})_{6}\right]^{3-/ 4-}$ as a redox probe and $\mathrm{KCl}$ as a supporting electrolyte $[17,20,37,41,43,45,47,53,56,57,59,63-65,69,80,85,87,90,105,117,124,125,127,131,132,136,141-143,147]$, but $\left[\mathrm{Ru}\left(\mathrm{NH}_{3}\right)_{6}\right]^{3+/ 2+}$ was also used [38].

$\mathrm{CV}$ carried out at different scan rates was most often employed to calculate the electroactive surface area $\left(\mathrm{A}_{\mathrm{ea}}\right)$ of bare or different modified electrodes while considering the slope of the regression equation corresponding to the $I_{p}=f\left(v^{1 / 2}\right)$ plot, where $I_{p}(A)$ repre- 
sents the peak current and $\mathrm{v}$ is the potential scan rate $(\mathrm{V} / \mathrm{s})$ $[16,20,38,39,41,43-49,53,56,59,64,67,72,74,82,85,87,88,106,124-126,128,141]$.

Chronoamperometry [68] and chronocoulometry were also used to assess the $\mathrm{A}_{\mathrm{ea}}$ of various working electrodes [53,62,143].

Generally, the electrode's electroactive surface area is higher than the geometrical one due to the porosity [40] or irregular surface morphologies. Moreover, the immobilization of various types of modifiers on the PGE surface resulted in an enhanced electroactive surface area $[17,63,69,85,110,136,141]$. For example, the surface areas of the AuNPs/P(Lmet)/PGE [130] and of the rGO/PWA/PGE [64] were about twofold higher than that of the corresponding bare PGE. The graphite lead modification with f-MWCNTs and AuNPs resulted in a specific electroactive surface that was 6.66 times larger [61], and the CNDs/MIP(PABSA)/PGE developed for folic acid determination presented an electroactive surface area enhanced 9.26 times vs. the bare PGE [123]. Likewise, $A_{\text {ea }}$ increased in the following order: PGE $\left(1.121 \mathrm{~cm}^{2}\right), \beta-C D-G / P G E\left(2.578 \mathrm{~cm}^{2}\right), \mathrm{Pt} / \beta-C D-G / P G E$ $\left(3.420 \mathrm{~cm}^{2}\right)$ and PGE $\left(15.29 \mathrm{~cm}^{2}\right), \mathrm{P}(\mathrm{SA}) \mathrm{PGE}\left(17.93 \mathrm{~cm}^{2}\right)$, NiONPs /PGE $\left(22.25 \mathrm{~cm}^{2}\right)$, and NiONPs/P(SA)/PGE $\left(35.45 \mathrm{~cm}^{2}\right)$ in the case of the modified PGEs recently developed for vitamin B6 [144] and avanafil and nimodipine [151] analysis, respectively.

$\mathrm{CV}$ using a redox active probe was applied to perform comparative studies of the electrochemical properties of various electrodes and to monitor the whole processes in the stepwise preparation of modified PGEs [36,38,49,67,108,123,126]. The performances of different types of electrodes were compared while considering the potential difference $\left(\Delta \mathrm{E}_{\mathrm{p}}\right)$ between the anodic and cathodic peak potentials and the peak currents. A decrease in $\Delta \mathrm{E}$ suggests faster electron transfer rates and therefore a more reversible electrode process, while larger peak currents indicate an enhanced $\mathrm{A}_{\text {ea }}$ and better electrocatalytic activity of the electrode $[10,45-47,72,109,131]$. Most studies revealed that PGE modification resulted in improved performance characteristics $[37,85,128,136,141,147]$, such as a decrease in $\Delta \mathrm{E}_{\mathrm{p}}$ from $440 \mathrm{mV}$ for the PGE to $80 \mathrm{mV}$ for the Chti/Sn/PGE [69] or improved electrochemical properties in the order of PGE < SNDGr/PGE < Cu-MOF/SNDGr/PGE due to enhanced peak currents and lowered $\Delta \mathrm{E}_{\mathrm{p}}[63]$.

EIS enables accurate assessment of the electrode resistance changes during the different successive modification steps $[38,69,126,127]$ and provides information about their interfacial electron transfer properties by interpreting the impedance spectrum (Nyquist plot). The Nyquist plot consists of a linear part at lower frequencies, which corresponds to the diffusion process, and a semicircle at higher frequencies, whose diameter can be used to calculate the charge transfer resistance at the electrode's surface (Rct). The charge transfer resistance of the electrode is obtained from a known equivalent circuit model (Randle's equivalent circuit) $[10,44,45,47,56,68,87,111,117,124]$. Thus, the larger semicircle indicates a high interfacial electron transfer resistance, whereas a small semicircle indicates a lower resistance, increased conductivity, and faster electron transfer. Usually, the ET [43,56,80] or modified electrodes employed $[20,36,37,45-47,53,57,59,61,63-$ $65,67,68,85,90,92,105,110,113,117,130,132,136,141-144,147,148]$ in pharmaceutical quantification presented a much lower Rct and thus a higher electron transfer capability in comparison with the bare PGE or the electrodes modified during the different steps of the manufacturing process. For example, EIS measurements emphasized that by covering the PGE with MIP/sol-gel, the Rct increased due to the surface passivation and decreased after potentiostatic deposition of N-doped graphene [127], AuNPs [91], or phosphotungstic acid/reduced graphene oxide (PWA/rGO) [65] due to the electronconducting properties of the nanomaterials. In the case of fabrication of the AuNPs@fCNFs/PGE for febuxostate and of the poly(SBT)/N-CNDs/CoNPs/PGE for donezepil quantification, the EIS results showed that the conductivity of the stepwise prepared electrodes increased in the order PGE < AuNPs/PGE < f-CNFs/PGE < AuNPs@f-CNFs/PGE [17] and $\mathrm{PGE}<\operatorname{poly}(\mathrm{SBT}) / \mathrm{PGE}<\operatorname{poly}(\mathrm{SBT}) / \mathrm{N}-\mathrm{CNDs} / \mathrm{PGE}<$ poly$(\mathrm{SBT}) / \mathrm{N}-\mathrm{CNDs} / \mathrm{CoNPs} / \mathrm{PGE}$ [131], respectively. 


\subsection{PGE Surface Characterization by Techniques of Electron Microscopy}

The modifications of the PGEs either in the final form or during the intermediate steps involving the deposition of various materials $[53,60,69,85,86,127,130,131,143]$ must be proven by surface analysis using non-destructive techniques like scanning electron microscopy (SEM) $[41,46,52,60,67,87,88,106,119,124,127,133,144-146,151]$, field effect SEM (FESEM) $[108,110,128,134,142,150]$, transmission electron microscopy (TEM) [52,87,134,144,146], and atomic force microscopy (AFM) $[36,37,109,110]$. These techniques, either alone or in combination, give valuable information about the surface morphology (e.g., texture and distribution of the materials $[50,62,65,72,90,103,117,132])$, topography (e.g., shape and size of the modifier(s) $[20,51,53,68,86,115,123,126,135,147])$, and roughness $[89,123,140]$.

Most SEM [47,56,57,62-64,80,85,111-113,127,130,136], FE-SEM [10,53], and AFM images $[10,110]$ revealed that the PGE had a smooth surface, but some research emphasized a flake-like structure $[20,54,55,58,60,69,89]$, whereas others indicated that the PGE surface contained pores $[46,61,141]$. On the other hand, SEM images showed that in the electrochemically pretreated PGEs, the graphite layers were broken into smaller pieces, and the effective surface area was increased $[10,36,56,62]$. The surface analysis techniques showed that the roughness, and consequently the effective surface area of the PGE, was increased by electrode modification $[37,63,64,135,137,141,147,148]$.

\subsection{PGE Surface Characterization by Spectroscopic Techniques Using X-rays}

Spectroscopic techniques involving $\mathrm{X}$-rays are often used in association with the electron microscopic techniques for the characterization of different materials. Energydispersive X-ray analysis (EDX or EDS) is an elemental analysis technique giving information about the chemical composition of a material, so this was frequently applied in the PGE modification process to reveal the elemental distribution by mapping each component $[10,17,20,48,87,110,120,124,126,131,132,134,146,148,150]$, thus confirming the presence of the modifier(s) on the electrode surface in different steps $[38,49,52,53,61-63,68,69,130,142,143,145,147]$. X-ray photoelectron spectroscopy (XPS), also known as electron spectroscopy for chemical analysis (ESCA), which provides information about the chemical composition of the material and the chemical state of the contained elements was sometimes used as a complementary technique to confirm the existence of certain materials at the PGE's surface $[10,128,134,144]$. X-ray diffraction (XRD) analysis was applied to gain structural information (crystallinity) about bare or modified PGEs. XRD analysis of bare PGEs confirmed that the main component was graphite, and it had good crystallinity [54,55]. In the case of modified PGEs, the XRD patterns were helpful in structural characterization of the prepared materials before and after their deposition at the electrode's surface $[17,62,63,108,110,124,134-136,144,146,148,149]$.

\subsection{PGE Surface Characterization by Optical Spectroscopic Techniques}

Fourier-Transform infrared (FT-IR) spectroscopy is a widely used technique for providing information about the chemical structure and bonds of organic, polymeric $[59,111,140]$, and inorganic materials like graphene-based ones [64,90,108,124,127,134,135,147,148,150]. Therefore, it was frequently employed to investigate the functional groups in different steps of the synthesis of modified PGEs in order to monitor the changes (e.g., immobilization of chemical species) that occurred at the electrode's surface during the fabrication process $[17,56,59,62,69,111,112,125,131-133,144,146,151]$. Binding mechanisms between the monomer(s) and the template(s) were suggested based on the comparative analysis of the FT-IR spectra of the monomer(s), template(s), MIP-adduct(s), and MIP(s) [48-52,126,128]. Some papers reported the Raman spectroscopic analysis of PGEs modified with graphenebased materials $[64,127,142]$, while UV-Vis spectra were useful in confirming the synthesis of AgNPs [146], CNDs [38,123,131], and [Ni(salen)] [149]. 


\section{Conclusions}

The examples presented in this review regarding PGE use in the voltametric analysis of pharmaceuticals emphasize the versatility of pencil graphite leads as working electrodes, because they are disposable and cheap (e.g., USD 0.01 per electrode [40] or EUR 0.06 vs. the disposable screen-printed graphite electrodes (EUR 2.7) [18]), they have high availability, being commercialized in common bookstores, and very importantly, they present low background currents and can be modified easier in comparison with other usually employed electrodes like GCEs or diamond electrodes [64]. Unmodified PGEs are user- and eco-friendly, while modified PGEs present better performance characteristics like selectivity and sensitivity, but they require more reagents and time for the whole analysis due to the various synthesis steps and supplementary investigations for surface characterization. All these and other important advantages offered by the PGE, either bare or as support for modified sensors, resulted in ever increasing interest in its use in the electrochemical analysis of various organic or inorganic species from very different matrices. According to the database provided by Scopus [155], in 1931, 1968, and 1989, only one paper referring to PGEs was published in each of these years. In 2016, the number increased to 94 , while in the last 2 years, the specialized literature has been enriched annually with over 150 reports on this topic. The increasing tendency seems to continue, as in the first month of 2022, 25 studies using PGEs in pharmaceutical analysis had already appeared. It must be emphasized that this brief statistic comprises all types of electrochemical sensors (voltametric, impedimetric, potentiometric, and photoelectrochemical) based on PGEs. Due to the huge number of papers existing on this subject, we chose to discuss in this review strictly the voltametric sensors based on PGEs developed in the last 10 years for the analysis of drugs. We considered that this is an important research domain due to three main aspects: (1) the pharmaceutical industry is developing very fast, and it is imperative to have rapid and simple tools for the quality control of intermediate and final products during the fabrication process; (2) drugs must be carefully administrated due to their possible side effects, and their concentrations in the body fluids must be precise and easily monitored; and (3) some pharmaceuticals may enter through different paths into the food chain or environment, and therefore their levels in such samples must be accurately controlled by affordable means. From all the instrumental methods, the electrochemical ones are among the cheapest, simplest, and most rapid ones. The instrumentation may be portable and simple to operate but provide the required sensitivity and selectivity of the analysis. The literature data and our personal research experience emphasized that the use of disposable PGEs as working electrodes in voltametric measurements offers many advantages, making them versatile tools for pharmaceutical analysis as they can be easily modified, resulting in more selective or selective sensors. In the literature, there are some reviews regarding PGE applications, but they are more general. This paper is a comprehensive review of PGEs, focusing on their applications in drug voltametric analysis. We tried to bring together and discuss almost all information published in the last 10 years on this topic, emphasizing the most relevant aspects, like PGE characteristics, surface modification procedures, and characterization techniques, but most importantly, actual examples of voltametric methods using PGEs for the analysis of pharmaceutically important compounds, pointing out the performance parameters of each method and the investigated samples.

Author Contributions: Conceptualization and methodology, I.G.D. and E.E.I.; resources and data curation, I.G.D. and G.O.T.; writing-original draft preparation, I.G.D., M.B., D.E.P., M.C.C. and V.D.; writing-review and editing, E.E.I. and G.O.T. All authors have read and agreed to the published version of the manuscript.

Funding: This research received no external funding.

Institutional Review Board Statement: Not applicable.

Informed Consent Statement: Not applicable.

Data Availability Statement: Not applicable. 
Conflicts of Interest: The authors declare no conflict of interest.

\section{References}

1. Council of Europe. European Pharmacopoeia; Council of Europe: Strasbourg, France, 2001.

2. Tasic, Z.Z.; Petrovic Mihajlovic, M.B.; Simonovic, A.T.; Radovanovic, M.B.; Antonijevi, M.M. Review of applied surface modifications of pencil graphite electrodes for paracetamol sensing. Results Phys. 2022, 22, 103911. [CrossRef]

3. David, I.G.; Litescu, S.C.; Popa, D.E.; Buleandra, M.; Iordache, L.; Albu, C.; Alecu, A.; Penu, R.L. Voltammetric analysis of naringenin at a disposable pencil graphite electrode-Application to polyphenol content determination in citrus juice. Anal. Methods 2018, 10, 5763-5772. [CrossRef]

4. David, I.G.; Oancea, A.G.; Buleandra, M.; Popa, D.E.; Iorgulescu, E.E.; Ciobanu, A.M. Disposable pencil graphite electrode for diosmin voltammetric analysis. Micromachines 2021, 12, 351. [CrossRef] [PubMed]

5. David, I.G.; Numan, N.; Buleandra, M.; Popa, D.E.; Lițescu, S.C.; Riga, S.; Ciobanu, A.M. Rapid voltammetric screening method for the assessment of bioflavonoid content using the disposable bare pencil graphite electrode. Chemosensors 2021, 9, 323. [CrossRef]

6. Buleandra, M.; Popa, D.E.; David, I.G.; Bacalum, E.; David, V.; Ciucu, A.A. Electrochemical behavior study of some selected phenylurea herbicides at activated pencil graphite electrode. Electrooxidation of linuron and monolinuron. Microchem. J. 2019, 147, 1109-1116. [CrossRef]

7. Wong, A.; Santos, A.M.; da Fonseca Alves, R.; Campanha Vicentini, F.; Fatibello-Filho, O.; Taboada Sotomayor, M.D.P. Simultaneous determination of direct yellow 50, tryptophan, carbendazim, and caffeine in environmental and biological fluid samples using graphite pencil electrode modified with palladium nanoparticles. Talanta 2021, 222, 121539. [CrossRef]

8. Sharma, S.; Jain, R.; Nitin Raja, A. Review-Pencil graphite electrode: An emerging sensing material. J. Electrochem. Soc. 2020, 167, 037501. [CrossRef]

9. Dilgin, D.G.; Karakaya, S. Differential pulse voltammetric determination of acyclovir in pharmaceutical preparations using a pencil graphite electrode. Mater. Sci. Eng. C 2016, 63, 570-576. [CrossRef] [PubMed]

10. Dokur, E.; Gorduk, O.; Sahin, Y. Cost-effective and facile production of a phosphorus doped graphite electrode for the electrochemical determination of pyridoxine. Electroanalysis 2021, 33, 1657-1667. [CrossRef]

11. Naik, K.M.; Ashi, C.R.; Nandibewoor, S.T. Anodic voltammetric behavior of hydroxyurea and its electroanalytical determination in pharmaceutical dosage form and urine. J. Electroanal. Chem. 2015, 755, 109-114. [CrossRef]

12. Available online: https://eur-lex.europa.eu/legal-content/EN/TXT/PDF/?uri=CELEX:32017R0852\&rid=7 (accessed on 25 December 2021).

13. Altunkaynak, Y.; Yavuz, O.; Levent, A. Firstly electrochemical examination of vildagliptin at disposable graphite sensor: Sensitive determination in drugs and human urine by square-wave voltammetry. Microchem. J. 2021, 170, 106653. [CrossRef]

14. David, I.G.; Popa, D.E.; Buleandra, M. Pencil graphite electrodes: A versatile tool in electroanalysis. J. Anal. Methods Chem. 2017, 2017, 1905968. [CrossRef]

15. Stradolini, F.; Kilic, T.; Di Consiglio, A.; Ozsoz, M.; De Micheli, G.; Carrara, S. Long-term monitoring of propofol and fouling effect on pencil graphite electrodes. Electroanalysis 2018, 30, 1363-1369. [CrossRef]

16. Purushothama, H.T.; Arthoba Nayaka, Y.; Vinay, M.M.; Manjunatha, P.; Yathisha, R.O.; Basavarajappa, K.V. Pencil graphite electrode as an electrochemical sensor for the voltammetric determination of chlorpromazine. J. Sci. Adv. Mater. Devices 2018, 3, 161-166. [CrossRef]

17. Ibrahim, H.; Temerk, Y. A novel electrochemical sensor based on gold nanoparticles decorated functionalized carbon nanofibers for selective determination of xanthine oxidase inhibitor febuxostat in plasma of patients with gout. Sens. Actuators B Chem. 2021, 347, 130626. [CrossRef]

18. Trnkova, L.; Triskova, I.; Cechal, J.; Farka, Z. Polymer pencil leads as a porous nanocomposite graphite material for electrochemical applications: The impact of chemical and thermal treatments. Electrochem. Commun. 2021, 126, 107018. [CrossRef]

19. Saleh, G.A.; Askal, H.F.; Refaat, I.H.; Naggar, A.H.; Abdel-aal, F.A.M. Adsorptive square wave voltammetric determination of the antiviral drug valacyclovir on a novel sensor of copper microparticles-modified pencil graphite electrode. Arab. J. Chem. 2016, 9, 143-151. [CrossRef]

20. Kouchakinejad, S.; Babaee, S.; Roshani, F.; Kouchakinejad, R.; Shirmohammadi, N.; Kaki, S. The performance of the new modified pencil graphite electrode in quantifying of insulin. Chem. Phys. Lett. 2020, 759, 137987. [CrossRef]

21. Dede, E.; Saglam, Ö.; Dilgin, Y. Sensitive voltammetric determination of niclosamide at a disposable pencil graphite electrode. Electrochim. Acta 2014, 127, 20-26. [CrossRef]

22. Temerk, Y.M.; Ibrahim, H.S.M.; Schuhmann, W. Square wave cathodic adsorptive stripping voltammetric determination of the anticancer drugs flutamide and irinotecan in biological fluids using renewable pencil graphite electrodes. Electroanalysis 2016, 28, 372-379. [CrossRef]

23. Dinc, E.; Dermiş, S.; Can Akcasoy, S.; Ceren Ertekin, Z. A New Chemometric strategy in electrochemical method optimization for the quantification of cefdinir in tablets, effervescent tablets and suspension samples. Electroanalysis 2020, 32, 613-619. [CrossRef]

24. Rizk, M.; Hendawy, H.A.M.; Abou El-Alamin, M.M.; Moawad, M.I. Sensitive anodic voltammetric determination of methylergometrine maleate in bulk and pharmaceutical dosage forms using differential pulse voltammetry. J. Electroanal. Chem. 2015, 749, 53-61. [CrossRef] 
25. Rizk, M.; Abou El-Alamin, M.A.; Hendawy, H.A.M.; Moawad, M.I. Highly sensitive differential pulse and square wave voltammetric methods for determination of strontium ranelate in bulk and pharmaceutical dosage form. Electroanalysis 2016, 28, 770-777. [CrossRef]

26. Shalaby, A.; Hassan, W.S.; Hendawy, H.A.M.; Ibrahim, A.M. Electrochemical oxidation behavior of itraconazole at different electrodes and its anodic stripping determination in pharmaceuticals and biological fluids. J. Electroanal. Chem. 2016, 763, 51-62. [CrossRef]

27. David, I.G.; Iordache, L.; Popa, D.E.; Buleandra, M.; David, V.; Iorgulescu, E.E. Novel voltammetric investigation of dipyridamole at disposable pencil graphite electrode. Turk. J. Chem. 2019, 43, 1109-1122. [CrossRef]

28. David, I.G.; Panait, A.L.; Buleandra, M.; Popa, D.E.; Cheregi, M.C. Simple voltammetric analysis of sulfamethoxazole at a disposable pencil graphite electrode. Rev. Roum. Chim. 2022. accepted.

29. David, I.G.; Buleandră, M.; Popa, D.-E.; Bercea, A.-M.; Ciucu, A.-A. Simple electrochemical chloramphenicol assay at a disposable pencil graphite electrode by square wave voltammetry and linear sweep voltammetry. Anal. Lett. 2021, 1-18. [CrossRef]

30. David, I.G.; Gâsnac, M.G.; Buleandră, M.; Popa, D.E. Simple and fast square wave voltammetric method for histamine H2-receptor antagonist famotidine quantification. Rev. Roum. Chim. 2021, 66, 573-578. [CrossRef]

31. David, I.G.; Popa, D.E.; Calin, A.-A.; Buleandră, M.; Iorgulescu, E.E. Voltammetric determination of famotidine on a disposable pencil graphite electrode. Turk. J. Chem. 2016, 40, 125-135. [CrossRef]

32. Sağlam, Ö.; Dilgin, D.G.; Ertek, B.; Dilgin, Y. Differential pulse voltammetric determination of eugenol at a pencil graphite electrode. Mater. Sci. Eng. C 2016, 60, 156-162. [CrossRef]

33. David, I.G.; Florea, M.-A.; Cracea, O.G.; Popa, D.E.; Buleandra, M.; Iorgulescu, E.E.; David, V.; Badea, I.A.; Ciucu, A.A Voltammetric Determination of vitamin B1 and vitamin B6 on a disposable electrode. Chem. Pap. 2015, 69, 901-910. [CrossRef]

34. Said, M.I.; Abdel-aal, F.A.M.; Rageh, A.H. Novel sponge-like Mn5O8 nanoparticles deposited on graphite electrode for electrochemical study of hepatitis C antiviral drug, elbasvir. Microchem. J. 2020, 157, 105056. [CrossRef]

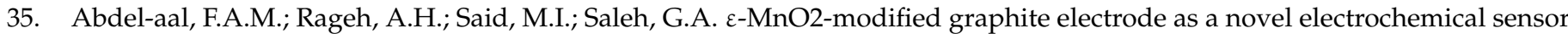
for the ultrasensitive detection of the newly FDA approved Hepatitis C antiviral drug ledipasvir. Anal. Chim. Acta 2018, 1038, 29-40. [CrossRef]

36. Rezaei, B.; Boroujeni, M.K.; Ensafi, A.A. Caffeine electrochemical sensor using imprinted film as recognition element based on polypyrrole, sol-gel, and gold nanoparticles hybrid nanocomposite modified pencil graphite electrode. Biosens. Bioelectron. 2014, 60, 77-83. [CrossRef] [PubMed]

37. Rezaei, B.; Boroujeni, M.K.; Ensafi, A.A. A novel electrochemical nanocomposite imprinted sensor for the determination of lorazepam based on modified polypyrrole@sol-gel@gold nanoparticles/pencil graphite electrode. Electrochim. Acta 2014, 123, 332-339. [CrossRef]

38. Pandey, I.; Jha, S.S. Molecularly imprinted polyaniline-ferrocene-sulfonic acid-carbon dots modified pencil graphite electrodes for chiral selective sensing of D-Ascorbic acid and L-Ascorbic acid: A clinical biomarker for preeclampsia. Electrochim. Acta 2015, 182, 917-928. [CrossRef]

39. Jadon, N.; Jain, R.; Pandey, A. Electrochemical analysis of amlodipine in some pharmaceutical formulations and biological fluid using disposable pencil graphite electrode. J. Electroanal. Chem. 2017, 788, 7-13. [CrossRef]

40. Alves, G.F.; Lisboa, T.P.; de Faria, L.V.; de Farias, D.M.; Matos, M.A.C.; Matos, R.C. Disposable pencil graphite electrode for ciprofloxacin determination in pharmaceutical formulations by square wave voltammetry. Electroanalysis 2021, 33, 543-549. [CrossRef]

41. Pinyou, P.; Blay, V.; Chansaenpak, K.; Lisnund, S. Paracetamol sensing with a pencil lead electrode modified with carbon nanotubes and polyvinylpyrrolidone. Chemosensors 2020, 8, 133. [CrossRef]

42. de Macêdo, I.Y.L.; Fernandes Alecrim, M.; Oliveira Neto, J.R.; Sapateiro Torres, I.M.; Vieira Thomaz, D.; de Souza Gil, E. Piroxicam voltammetric determination by ultra low cost pencil graphite electrode. Braz. J. Pharm. Sci. 2020, 56, e17344. [CrossRef]

43. Purushothama, H.T.; Arthoba Nayaka, Y. Electrochemical study of hydrochlorothiazide on electrochemically pretreated pencil graphite electrode as a sensor. Sens. Bio-Sens. Res. 2017, 16, 12-18. [CrossRef]

44. Purushothama, H.T.; Arthoba Nayaka, Y. Pencil graphite electrode based electrochemical system for the investigation of antihypertensive drug hydrochlorothiazide: An electrochemical study. Chem. Phys. Lett. 2019, 734, 136718. [CrossRef]

45. Vinay, M.M.; Nayaka, Y.A.; Yatisha, R.O.; Basavarajappa, K.V.; Manjunatha, P.; Purushothama, H.T. Development of Azure-B modified pencil graphite electrode as an electrochemical sensor for the investigation of levofloxacin in pharmaceutical and biological samples. Chem. Data Collect. 2020, 28, 100441. [CrossRef]

46. Mohamed, A.-M.I.; Rageh, A.H.; Abdel-aal, F.A.M.; Ali, A.-M.B.H. Pencil graphite electrode decorated with xylenol orange flakes for studying possible pharmacokinetic interaction between vardenafil and daclatasvir. Electroanalysis 2020, 32, 635-647. [CrossRef]

47. Manjunatha, P.; Arthoba Nayaka, Y.; Chethana, B.K.; Vidyasagar, C.C.; Yathisha, R.O. Development of multi-walled carbon nanotubes modified pencil graphite electrode for the electrochemical investigation of aceclofenac present in pharmaceutical and biological samples. Sens. Bio-Sens. Res. 2018, 17, 7-17. [CrossRef]

48. Prasad, B.B.; Kumar, A.; Singh, K. Molecularly imprinted polymer-based electrochemical sensor using functionalized fullerene as a nanomediator for ultratrace analysis of primaquine. Carbon 2016, 109, 196-207. [CrossRef]

49. Prasad, B.B.; Singh, R.; Kumar, A. Development of imprinted polyneutral red/electrochemically reduced graphene oxide composite for ultra-trace sensing of 6-thioguanine. Carbon 2016, 102, 86-96. [CrossRef] 
50. Prasad, B.B.; Prasad, A.; Prasad Tiwari, M.; Madhuri, R. Multiwalled carbon nanotubes bearing 'terminal monomeric unit' for the fabrication of epinephrine imprinted polymer-based electrochemical sensor. Biosens. Bioelectron. 2013, 45, 114-122. [CrossRef] [PubMed]

51. Prasad, B.B.; Fatma, S. One MoNomer doubly imprinted dendrimer nanofilm modified pencil graphite electrode for simultaneous electrochemical determination of norepinephrine and uric acid. Electrochim. Acta 2017, 232, 474-483. [CrossRef]

52. Prasad, B.B.; Singh, R.; Singh, K. Development of highly electrocatalytic and electroconductingimprinted film using Ni nanomer for ultra-trace detection of thiamine. Sens. Actuators B Chem. 2017, 246, 38-45. [CrossRef]

53. Dehnavi, A.; Soleymanpour, A. Silver nanoparticles/poly(L-cysteine) nanocomposite modified pencil graphite for selective electrochemical measurement of guaifenesin in real samples. Measurement 2021, 175, 109103. [CrossRef]

54. Rejithamol, R.; Keerthi, P.R.; Beena, S. A novel disposable pencil graphite electrode for the voltammetric determination of cysteamine. Mater. Today Proc. 2019, 18, 5081-5086.

55. Krishnan, R.G.; Greeshma, S.; Shivon, D.M.; Suryasree, S.R.; Beena, S. Morphological studies of disposable graphite and its effective utilization for vitamin B12 analysis in pharmaceutical formulations. Mater. Today Proc. 2019, 18, 3314-3320. [CrossRef]

56. Karaboduk, K. Development of a voltammetric method for the determination of rapamycin in pharmaceutical samples at pretreated pencil graphite electrode. J. Chin. Chem. Soc. 2021, 68, 1722-1730. [CrossRef]

57. Fard, G.P.; Alipour, E.; Sabzi, R.E.A. Modification of a disposable pencil graphite electrode with multiwalled carbon nanotubes: Application to electrochemical determination of diclofenac sodium in some pharmaceutical and biological samples. Anal. Methods 2016, 8, 3966-3974. [CrossRef]

58. Alipour, E.; Majidi, M.R.; Hoseindokht, O. Development of simple electrochemical sensor for selective determination of methadone in biological samples using multi-walled carbon nanotubes modified pencil graphite electrode. J. Chin. Chem. Soc. 2015, 62, 461-468. [CrossRef]

59. Tabanlıgil Calam, T. A modified pencil graphite electrode with 2-thiobarbituric acid for the efficient and cheap voltammetric sensing of 4-aminophenol in water samples and child syrup sample. J. Food Compos. Anal. 2021, 98, 103809. [CrossRef]

60. Rostami-Javanroudi, S.; Babakhanian, A. New electrochemical sensor for direct quantification of vitamin K in human blood serum. Microchem. J. 2021, 163, 105716. [CrossRef]

61. Kolahi-Ahari, S.; Deiminiat, B.; Rounaghi, G.H. Modification of a pencil graphite electrode with multiwalled carbon nanotubes capped gold nanoparticles for electrochemical determination of tramadol. J. Electroanal. Chem. 2020, 862, 113996. [CrossRef]

62. Pashazadeh, S.; Habibi, B. Determination of isoniazid by a copper-based metal-organic frameworks/carbon nitride quantum dots modified pencil graphite electrode as a highly sensitive and selective sensor. J. Electroanal. Chem. 2020, 876, 114493. [CrossRef]

63. Habibi, B.; Pashazadeh, S.; Saghatforoush, L.A.; Pashazadeh, A. Direct electrochemical synthesis of the copper based metal-organic framework on/in the heteroatoms doped graphene/pencil graphite electrode: Highly sensitive and selective electrochemical sensor for sertraline hydrochloride. J. Electroanal. Chem. 2021, 888, 115210. [CrossRef]

64. Oghli, H.; Soleymanpour, A. Polyoxometalate/reduced graphene oxide modified pencil graphite sensor for the electrochemical trace determination of paroxetine in biological and pharmaceutical media. Mater. Sci. Eng. C 2020, 108, 110407. [CrossRef] [PubMed]

65. Oghli, H.; Soleymanpour, A. Ultrasensitive electrochemical sensor for simultaneous determination of sumatriptan and paroxetine using molecular imprinted polymer/sol-gel/ polyoxometalate/rGO modified pencil graphite electrode. Sens. Actuators B Chem. 2021, 344, 130215. [CrossRef]

66. Ibrahim, H.; Temerk, Y. A novel disposable electrochemical sensor based on modifying graphite pencil lead electrode surface with nanoacetylene black for simultaneous determination of antiandrogens flutamide and cyproterone acetate. J. Electroanal. Chem. 2020, 859, 113836. [CrossRef]

67. Zabardasti, A.; Afrouzi, H.; Talemi, R.P. A simple and sensitive methodology for voltammetric determination of valproic acid in human blood plasma samples using 3-aminopropyletriethoxy silane coatedmagnetic nanoparticles modified pencil graphite electrode. Mater. Sci. Eng. C 2017, 76, 425-430. [CrossRef]

68. Sisolakova, I.; Hovancova, J.; Orinakova, R.; Orinak, A.; Garcia, D.R.; Shylenko, O.; Radonak, J. Comparison of insulin determination on NiNPs/chitosan-MWCNTs and NiONPs/chitosan-MWCNTs modified pencil graphite electrode. Electroanalysis 2019, 31, 103-112. [CrossRef]

69. Nagarajan, S.; Vairamuthu, R. Electrochemical detection of riboflavin using tin-chitosan modified pencil graphite electrode. $J$. Electroanal. Chem. 2021, 891, 115235. [CrossRef]

70. Deepa, S.; Kumara Swamy, B.E.; Vasantakumar Pai, K. Voltammetric detection of anticancer drug doxorubicin at pencil graphite electrode: A voltammetric study. Sens. Int. 2020, 1, 100033. [CrossRef]

71. Yen, P.T.H.; Anh, N.H.; Ha, V.T.T.; Hung, L.Q.; Phong, P.H.; Hien, C.T.T. Electrochemical properties of amoxicillin on an economical, simple graphite pencil electrode and the ability of the electrode in amoxicillin detection. Vietnam J. Chem. 2020, 58 , 201-205. [CrossRef]

72. Tigari, G.; Manjunatha, J.G. A surfactant enhanced novel pencil graphite and carbon nanotube composite paste material as an effective electrochemical sensor for determination of riboflavin. J. Sci. Adv. Mater. Dev. 2020, 5, 56-64. [CrossRef]

73. Aydin, I.; Akgun, H.; Talay Pınar, P. Analytical determination of the oxazolidinone antibiotic linezolid at a pencil graphite and carbon paste electrodes. ChemistrySelect 2019, 4, 9966-9971. [CrossRef] 
74. Sawkar, R.R.; Patil, V.B.; Shanbhag, M.M.; Shetti, N.P.; Tuwar, S.M.; Aminabhavi, T.M. Detection of ketorolac drug using pencil graphite electrode. Biomed. Eng. Adv. 2021, 2, 100009. [CrossRef]

75. Kojabad, R.N.; Ebrahimiasl, S. Pencil graphite electrode modified nanosensor for detection and determination of tramadol in blood serum. QUID Investig. Cienc. Tecnol. 2017, 1, 597-604.

76. Gowda, J.I.; Nandibewoor, S.T. Electrochemical behavior of 4-aminophenazone drug at a graphite pencil electrode and its application in real samples. Ind. Eng. Chem. Res. 2012, 51, 15936-15941. [CrossRef]

77. Gadallah, M.I.; Ali, H.R.H.; Askal, H.F.; Saleh, G.A. Development of terbium based sensor for determination of imipenem in dosage forms and real samples. J. Mol. Liq. 2016, 276, 705-713. [CrossRef]

78. Gowda, J.I.; Nandibewoor, S.T. Electrochemical characterization and determination of paclitaxel drug using graphite pencil electrode. Electrochim. Acta 2014, 116, 326-333. [CrossRef]

79. Buleandră, M.; Popa, D.E.; Popa, A.; Codreanu, N.A.M.; David, I.G. Multi-analyte sensor based on pencil graphite electrode for riboflavin and pyridoxine determination. J. Electrochem. Soc. 2022, 169, 017517. [CrossRef]

80. Parvizi-Fard, G.; Alipour, E.; Sefidi, P.Y.; Sabzi, R.E. Pretreated pencil graphite electrode as a versatile platform for easy measurement of diclofenac sodium in a number of biological and pharmaceutical samples. J. Chin. Chem. Soc. 2018, 65, 472-484. [CrossRef]

81. Koyun, O.; Gorduk, S.; Arvas, M.B.; Sahin, Y. Electrochemically treated pencil graphite electrodes prepared in one step for the electrochemical determination of paracetamol. Russ. J. Electrochem. 2018, 54, 796-808. [CrossRef]

82. Khashaba, P.Y.; Ali, H.R.H.; El-Wekil, M.M. Complexation based voltammetric determination of pantoprazole sodium in pharmaceutical formulations and rabbit plasma. Electroanalysis 2017, 29, 890-897. [CrossRef]

83. Rana, A.; Baig, N.; Saleh, T.A. Electrochemically pretreated carbon electrodes and their electroanalytical applications-A review. J. Electroanal. Chem. 2019, 833, 313-332. [CrossRef]

84. Levent, A.; Onal, G. Application of a pencil graphite electrode for voltammetric simultaneous determination of ascorbic acid, norepinephrine, and uric acid in real samples. Turk. J. Chem. 2018, 42, 460-471. [CrossRef]

85. Bayraktepe, D.E.; Yazan, Z. Application of single-use electrode based on nano-clay and MWCNT for simultaneous determination of acetaminophen, ascorbic acid and acetylsalicylic acid in pharmaceutical dosage. Electroanalysis 2020, 32, 1263-1272. [CrossRef]

86. Fouladgar, M.; Karimi-Maleh, H.; Opoku, F.; Govender, P.P. Electrochemical anticancer drug sensor for determination of raloxifene in the presence of tamoxifen using graphene-CuO-polypyrrole nanocomposite structure modified pencil graphite electrode: Theoretical and experimental investigation. J. Mol. Liq. 2020, 311, 113314. [CrossRef]

87. Hatamluyi, B.; Lorestani, F.; Es'haghi, Z. Au/Pd@rGO nanocomposite decorated with poly (L-Cysteine) as a probe forsimultaneous sensitive electrochemical determination of anticancer drugs, ifosfamide and etoposide. Biosens. Bioelectron. 2018, 120, 22-29. [CrossRef]

88. Hatamluyi, B.; Es'haghi, Z. A layer-by-layer sensing architecture based on dendrimer and ionic liquid supported reduced graphene oxide for simultaneous hollow-fiber solid phase microextraction and electrochemical determination of anti-cancer drug imatinib in biological samples. J. Electroanal. Chem. 2017, 801, 439-449. [CrossRef]

89. Yan, C.; Liu, X.; Zhang, R.; Chen, Y.; Wang, G. A selective strategy for determination of ascorbic acid based on molecular imprinted copolymer of o-phenylenediamine and pyrrole. J. Electroanal. Chem. 2016, 780, 276-281. [CrossRef]

90. Rouhani, M.; Soleymanpour, A. Ultrasensitive electrochemical determination of trace ceftizoxime using a thin film of Preyssler nanocapsules on pencil graphite electrode surface modified with reduced graphene oxide. Microchem. J. 2021, 165, 106160. [CrossRef]

91. Rezaei, B.; Lotfi-Forushani, H.; Ensafi, A.A. Modified Au nanoparticles-imprinted sol-gel, multiwall carbon nanotubes pencil graphite electrode used as a sensor for ranitidine determination. Mater. Sci. Eng. C 2014, 37, 113-119. [CrossRef] [PubMed]

92. Rezaei, B.; Foroughi-Dehnavi, S.; Ensafi, A.A. Fabrication of electrochemical sensor based on molecularly imprinted polymer and nanoparticles for determination trace amounts of morphine. Ionics 2015, 21, 2969-2980. [CrossRef]

93. Oghli, H.A.; Alipour, E.; Asadzadeh, M. Development of a novel voltammetric sensor for the determination of methamphetamine in biological samples on the pretreated pencil graphite electrode. RSC Adv. 2015, 5, 9674-9682. [CrossRef]

94. Alipour, E.; Gasemlou, S. Easy modification of pencil graphite electrode for discrimination and determination of morphine in biological and street samples. Anal. Methods 2012, 4, 2962-2969. [CrossRef]

95. Gowda, J.I.; Hurakadli, G.S.; Nandibewoor, S.T. Pretreated graphite pencil electrode based voltammetric sensing of albendazole. Anal. Chem. Lett. 2017, 7, 389-401. [CrossRef]

96. Buleandră, M.; Popa, D.E.; David, I.G.; Ciucu, A.A. A simple and efficient cyclic square wave voltammetric method for simultaneous determination of epinephrine and norepinephrine using an activated pencil graphite electrode. Microchem. J. 2021, 160, 105621. [CrossRef]

97. Buleandră, M.; Ciucu, A.A.; David, I.G.; Popa, D.E.; Ciobanu, A.M.; Ştefănescu, C.D. Simultaneous determination of epinephrine and norepinephrine by electrochemical reduction at the pre-treated pencil graphite electrode. Rev. Roum. Chim. 2021, 66, 567-572. [CrossRef]

98. Ciucu, A.A.; Buleandră, M.; Ciurea, T.; Stoica, V.N.; Ştefanescu, C.D.; Ciobanu, A. A new voltammetric approach for electrochemical determination of lamotrigine in pharmaceutical samples. Electroanalysis 2021, 33, 1389-1392. [CrossRef]

99. Mollarasouli, F.; Zor, E.; Ozcelikay, G.; Ozkan, S.A. Magnetic nanoparticles in developing electrochemical sensors for pharmaceutical and biomedical applications. Talanta 2021, 226, 122108. [CrossRef] [PubMed] 
100. Lawal, A.T. Progress in utilisation of graphene for electrochemical biosensors. Biosens. Bioelectron. 2018, 106, 149-178. [CrossRef]

101. Akanda, M.R.; Sohail, M.; Aziz, M.A.; Kawde, A.-N. Recent advances in nanomaterial-modified pencil graphite electrodes for electroanalysis. Electroanalysis 2016, 28, 408-424. [CrossRef]

102. Beluomini, M.A.; da Silva, J.L.; Cardoso de Sa, A.; Buffon, E.; Pereira, T.C.; Stradiotto, N.R. Electrochemical sensors based on molecularly imprinted polymer on nanostructured carbon materials: A review. J. Electroanal. Chem. 2019, 840, 343-366. [CrossRef]

103. Temerk, Y.; Ibrahim, M.; Ibrahim, H.; Kotb, M. Adsorptive stripping voltammetric determination of anticancer drug lomustine in biological fluids using in situ mercury film coated graphite pencil electrode. J. Electroanal. Chem. 2016, 760, 135-142. [CrossRef]

104. Asadpour-Zeynali, K.; Majidi, M.R.; Najafi-Marandi, P.; Norysaray, Z. Electrocatalytic reduction of metronidazole on bismuth modified pencil-lead electrode. J. Chin. Chem. Soc. 2013, 60, 1253-1259. [CrossRef]

105. Güzel, R.; Ekși, H.; Dinç, E.; Solak, A.O. Determination of acetaminophen in commercial formulations using silver nanostructured aniline modified pencil graphite electrode. J. Electrochem. Soc. 2013, 160, B119-B124. [CrossRef]

106. Nezhadali, A.; Mojarrab, M. Computational design and multivariate optimization of an electrochemical metoprolol sensor based on molecular imprinting in combination with carbon nanotubes. Anal. Chim. Acta 2016, 924, 86-98. [CrossRef]

107. Nezhadali, A.; Mojarrab, M. Fabrication of an electrochemical molecularly imprinted polymer triamterene sensor based on multivariate optimization using multi-walled carbon nanotubes. J. Electroanal. Chem. 2015, 744, 85-94. [CrossRef]

108. Pourbeyram, S.; Moosavifar, M.; Ashtari, L. Ultra-sensitive determination of insulin on pencil graphite electrode modified by cerium salen encapsulated zeolite (CS@Z-PGE). Microporous Mesoporous Mater. 2017, 242, 25-33. [CrossRef]

109. Pattar, V.P.; Nandibewoor, S.T. Staircase voltammetric determination of 2-thiouracil in pharmaceuticals and human biological fluids at polyaniline and polypyrrole film modified sensors. Sens. Actuators A Phys. 2016, 250, 40-47. [CrossRef]

110. Arabali, V.; Malekmohammadi, S.; Karimi, F. Surface amplification of pencil graphite electrode using CuO nanoparticle/polypyrrole nanocomposite; a powerful electrochemical strategy for determination of tramadol. Microchem. J. 2020, 158, 105179. [CrossRef]

111. Khashaba, P.Y.; Refat, H.; Ali, H.; El-Wekil, M.M. A new and cost effective approach for simultaneous voltammetric analysis of two related benzimidazole drugs and their determination in biological fluids. Electroanalysis 2017, 29, 1643-1650. [CrossRef]

112. Khashaba, P.Y.; Refat, H.; Ali, H.; El-Wekil, M.M. Simultaneous voltammetric analysis of anti-ulcer and D2-antagonist agents in binary mixture using redox sensor and their determination in human serum. Mater. Sci. Eng. C 2017, 75, 733-741. [CrossRef]

113. Gadallah, M.I.; Ali, H.R.H.; Askal, H.F.; Saleh, G.A. Poly (bromocresol green) flakes-decorated pencil graphite electrode for selective electrochemical sensing applications and pharmacokinetic studies. Mater. Sci. Eng. C 2019, 102, 634-645. [CrossRef]

114. Nezhadali, A.; Pirayesh, S.; Shadmehri, R. Computer-assisted sensor design and analysis of 2-aminobenzimidazole in biological model samples based on electropolymerized-molecularly imprinted polypyrrole modified pencil graphite electrode. Sens. Actuators B Chem. 2013, 185, 17-23. [CrossRef]

115. Nezhadali, A.; Mehri, L.; Shadmehri, R. Determination of benzimidazole in biological model samples using electropolymerizedmolecularly imprinted polypyrrole modified pencil graphite sensor. Sens. Actuators B Chem. 2012, 171-172, 1125-1131. [CrossRef]

116. Nezhadali, A.; Mehri, L.; Shadmehri, R. Determination of methimazole based on electropolymerized-molecularly imprinted polypyrrole modified pencil graphite sensor. Mater. Sci. Eng. C 2018, 85, 225-232. [CrossRef] [PubMed]

117. Tadi, K.K.; Motghare, R.V.; Ganesh, V. Electrochemical detection of sulfanilamide using pencil graphite electrode based on molecular imprinting technology. Electroanalysis 2014, 26, 2328-2336. [CrossRef]

118. Gürler, B.; Özkorucuklu, S.P.; Kir, E. Voltammetric behavior and determination of doxycycline in pharmaceuticals at molecularly imprinted and non-imprinted overoxidized polypyrrole electrodes. J. Pharm. Biomed. Anal. 2013, 84, 263-268. [CrossRef]

119. Koseoglu, T.S.; Durgut, A. Development of a novel molecularly imprinted overoxidized polypyrrole electrode for the determination of sulfasalazine. Electroanalysis 2020, 32, 2072-2081. [CrossRef]

120. Singh, K.; Prasad, B.B. Molecularly imprinted polymer-based core-shells (solid vs hollow) @pencil graphite electrode for electrochemical sensing of certain anti-HIV drugs. Sens. Actuators B Chem. 2017, 244, 167-174. [CrossRef]

121. Nezhadali, A.; Shadmehri, R. Neuro-genetic multi-objective optimization and computer-aided design of pantoprazole molecularly imprinted polypyrrole sensor. Sens. Actuators B Chem. 2014, 202, 240-251. [CrossRef]

122. Nezhadali, A.; Sadeghzadeh, S. Experimental design-artificial neural network-genetic algorithm optimization and computerassisted design of celecoxib molecularly imprinted polymer/carbon nanotube sensor. J. Electroanal. Chem. 2017, 795, 32-40. [CrossRef]

123. Güney, S. Electrochemical synthesis of molecularly imprinted poly(p-aminobenzene sulphonic acid) on carbon nanodots coated pencil graphite electrode for selective determination of folic acid. J. Electroanal. Chem. 2019, 854, 113518. [CrossRef]

124. Hatamluyi, B.; Es'haghi, Z. Electrochemical biosensing platform based on molecularly imprinted polymer reinforced by ZnOgraphene capped quantum dots for 6-mercaptopurine detection. Electrochim. Acta 2018, 283, 1170-1177. [CrossRef]

125. Singh, R.; Jaiswal, S.; Singh, K.; Fatma, S.; Prasad, B.B. Biomimetic polymer-based electrochemical sensor using methyl blueadsorbed reduced graphene qxide and functionalized multiwalled carbon nanotubes for trace sensing of cyanocobalamin. ACS Appl. Nano Mater. 2018, 1, 4652-4660. [CrossRef]

126. Prasad, B.B.; Pathak, P.K. Development of surface imprinted nanospheres using the inverse suspension polymerization method for electrochemical ultra sensing of dacarbazine. Anal. Chim. Acta 2017, 974, 75-86. [CrossRef] [PubMed] 
127. Oghli, A.H.; Soleymanpour, A. Pencil graphite electrode modified with nitrogen-doped graphene and molecular imprinted polyacrylamide/sol-gel as an ultrasensitive electrochemical sensor for the determination of fexofenadine in biological media. Biochem. Eng. J. 2021, 167, 107920. [CrossRef]

128. Kumar, A.; Pathak, P.K.; Prasad, B.B. Electrocatalytic imprinted polymer of N-doped hollow carbon nanosphere-palladium nanocomposite for ultratrace detection of anticancer drug 6-mercaptopurine. ACS Appl. Mater. Interfaces 2019, 11, 16065-16074. [CrossRef] [PubMed]

129. Nezhadali, A.; Bonakdar, G.A. Multivariate optimization of mebeverine analysis using molecularly imprinted polymer electrochemical sensor based on silver nanoparticles. J. Food Drug Anal. 2019, 27, 305-314. [CrossRef] [PubMed]

130. Bayraktepe, D.E.; Inal, E.K.; Yazan, Z. Preparation and characterization of a pencil graphite electrode modified with gold nanoparticles decorated poly (L-methionine) and its use in the simultaneous sensitive electrochemical analysis of ascorbic acid, acetaminophen, chlorpheniramine maleate, and caffeine. Microchem. J. 2021, 171, 106812. [CrossRef]

131. Mohamed, F.A.; Khashaba, P.Y.; Shahin, R.Y.; El-Wekil, M.M. Tunable ternary nanocomposite prepared by electrodeposition for biosensing of centrally acting reversible acetyl cholinesterase inhibitor donepezil hydrochloride in real samples. Colloids Surfaces A Physicochem. Eng. Asp. 2019, 567, 76-85. [CrossRef]

132. Dehnavi, A.; Soleymanpou, A. Titanium dioxide/multi-walled carbon nanotubes composite modified pencil graphite sensor for sensitive voltammetric determination of propranolol in real samples. Electroanalysis 2021, 33, 355-364. [CrossRef]

133. Es'haghi, Z.; Moeinpour, F. Carbon nanotube/polyurethane modified hollow fiber-pencil graphite electrode for in situ concentration and electrochemical quantification of anticancer drugs capecitabine and erlotinib. Eng. Life Sci. 2019, 19, 302-314. [CrossRef]

134. Das, T.R.; Jena, S.K.; Madhuri, R.; Sharma, P.K. Polymeric iron oxide-graphene nanocomposite as a trace level sensor of vitamin C. Appl. Surf. Sci. 2018, 449, 304-313. [CrossRef]

135. Senel, M.; Durmus, Z.; Alachkar, A. Measurement of the antipsychotic clozapine using reduced graphene oxide nanocomposites$\mathrm{Au} / \mathrm{Pd} /$ Pt electrodes. Electroanalysis 2021, 33, 1585-1595. [CrossRef]

136. Bayraktepe, D.E.; Yazan, Z.; Onal, M. Sensitive and cost effective disposable composite electrode based on graphite, nanosmectite and multiwall carbon nanotubes for the simultaneous trace level detection of ascorbic acid and acetylsalicylic acid in pharmaceuticals. Talanta 2019, 203, 131-139. [CrossRef] [PubMed]

137. Ensafi, A.A.; Khoddami, E.; Rezaei, B. A combined liquid three-phase micro-extraction and differential pulse voltammetric method for preconcentration and detection of ultra-trace amounts of buprenorphine using a modified pencil electrode. Talanta 2013, 116, 1113-1120. [CrossRef] [PubMed]

138. Izadyar, A.; Arachchige, D.R.; Cornwell, H.; Hershberger, J.C. Ion transfer stripping voltammetry for the detection of nanomolar levels of fluoxetine, citalopram, and sertraline in tap and river water samples. Sens. Actuators B Chem. 2016, 223 , 226-233. [CrossRef]

139. Dehghanzade, M.; Alipour, E. Voltammetric determination of diazepam using a bismuth modified pencil graphite electrode. Anal Methods 2016, 8, 1995-2004. [CrossRef]

140. Rezaeifar, Z.; Rounaghi, G.H.; Es'haghi, Z.; Chamsaz, M. Electrochemical determination of anticancer drug, flutamide in human plasma sample using a microfabricated sensor based on hyperbranchedpolyglycerol modified graphene oxide reinforced hollow fiber-pencil graphite electrode. Mater. Sci. Eng. C 2018, 91, 10-18. [CrossRef]

141. Manjunatha, P.; Nayaka, Y.A. Cetyltrimethylammonium bromide-gold nanoparticles composite modified pencil graphite electrode for the electrochemical investigation of cefixime present in pharmaceutical formulations and biology. Chem. Data Collect. 2019, 21, 100217. [CrossRef]

142. Azadmehr, F.; Zarei, K. Ultrasensitive determination of ceftizoxime using pencil graphite electrode modified by hollow gold nanoparticles/reduced graphene oxide. Arab. J. Chem. 2020, 13, 1890-1900. [CrossRef]

143. Akbari Hasanjani, H.R.; Zarei, K. Electrochemical sensor for ultrasensitive determination of ceftazidime using hollow platinum nanoparticles/reduced graphene oxide/pencil graphite electrode. Chem. Pap. 2018, 72, 1935-1944. [CrossRef]

144. Rison, S.; Mathew, A.T.; George, L.; Maiyalagan, T.; Hegde, G.; Varghese, A. Pt nanospheres decorated graphene- $\beta$-CD modified pencil graphite electrode for the electrochemical determination of vitamin B6. Top Catal. 2022. [CrossRef]

145. Sangeetha, N.S.; Narayanan, S.S. Effective electrochemical detection of riboflavin and butylated hydroxy anisole based on azure A and nickel hexacyanoferrate framework on graphite electrode. Chem. Data Collect. 2020, 30, 100544. [CrossRef]

146. Sangsefidi, F.S.; Salavati-Niasari, M.; Mazaheri, S.; Sabet, M. Controlled green synthesis and characterization of CeO2 nanostructures as materials for the determination of ascorbic acid. J. Mol. Liq. 2017, 241, 772-781. [CrossRef]

147. Rouhani, M.; Soleymanpour, A. Preparation of Dawson heteropolyacid-embedded silver nanoparticles/graphene oxide nanocomposite thin film used to modify pencil graphite electrode as a sensor for trace electrochemical sensing of levodopa. Mater. Sci. Eng. C 2020, 117, 111287. [CrossRef] [PubMed]

148. Dehnavi, A.; Soleymanpour, A. Highly sensitive voltammetric electrode for the trace measurement of methyldopa based on a pencil graphite modified with phosphomolibdate/graphene oxide. Microchem. J. 2020, 157, 104969. [CrossRef]

149. Martins, T.S.; Bott-Neto, J.L.; Raymundo-Pereira, P.A.; Ticianelli, E.A.; Machado, S.A.S. An electrochemical furosemide sensor based on pencil graphite surface modified with polymer film Ni-salen and $\mathrm{Ni}(\mathrm{OH}) 2 / \mathrm{C}$ nanoparticles. Sens. Actuators B Chem. 2018, 276, 378-387. [CrossRef]

150. Ensafi, A.A.; Khoddami, E.; Rezaei, B.; Jafari-Asl, M. A supported liquid membrane for microextraction of insulin, and its determination with a pencil graphite electrode modified with RuO2-graphene oxide. Mikrochim. Acta 2015, 182, 1599-1607. [CrossRef] 
151. Ali, A.-M.B.H.; Abdel-aal, F.A.M.; Rageh, A.H.; Mohamed, A.-M.I. Hybrid NiO nanostructured/sulfanilamide polymeric film for studying possible pharmacokinetic interaction between avanafil and nimodipine in real human serum by their simultaneous determination using square-wave voltammetry. Microchem. J. 2022, 172, 106895. [CrossRef]

152. Singh, K.; Jaiswal, S.; Singh, R.; Fatma, S.; Prasad, B.B. One-by-one imprinting in two eccentric layers of hollow core-shells: Sequential electroanalysis of anti-HIV drugs. Biosens. Bioelectron. 2018, 111, 82-89. [CrossRef]

153. Fakahari, A.R.; Sahragard, A.; Ahmar, H.; Tabani, H. A novel platform sensing based on combination of electromembrane-assisted solid phase microextraction with linear sweep voltammetry for the determination of tramadol. J. Electroanal. Chem. 2015, 747, 12-19. [CrossRef]

154. Riman, D.; Rozsypal, J.; Halouzka, V.; Hrbac, J.; Jirovsky, D. The use of micro carbon pencil lead electrode for sensitive HPLC-ED analysis of selected antipsychotic drugs. Microchem. J. 2020, 154, 104406. [CrossRef]

155. Available online: www.scopus.com (accessed on 5 February 2022). 